\title{
Braid group and anyons on a cylinder
}

\author{
Yasuhiro Hatsugai and Mahito Kohmoto \\ Institute for Solid State Physics, University of Tokyo, 7-22-1 Roppongi, Minato-ku, Tokyo 106, Japan \\ Yong-Shi Wu \\ Institute for Solid State Physics, University of Tokyo, 7-22-1 Roppongi, Minato-ku, Tokyo 106, Japan \\ and Department of Physics, University of Utah, Salt Lake City, Utah 84112
}

(Received 5 July 1990; revised manuscript received 18 October 1990)

\begin{abstract}
In this paper we present a careful reexamination of anyons on a cylinder (or annulus), starting from the braid-group analysis. Proper attention is paid to the topological features arising from the existence of noncontractible loops. The rule for putting anyons on a square lattice has to be modified when the periodic boundary condition is imposed on one direction. In contrast to the annulus, one extra restriction is needed for the cylinder geometry to recover its symmetry between the two edges. We have performed some finite-system calculations. The consistency of our results has been checked by the agreement from two sets of seemingly different rules and also by that with free fermions. We have also calculated the spectral flow of the excited states with varying statistics or flux and have seen a lot of level crossings, which seem to be a general feature of anyon systems. The mean-field treatment is found to be good until level crossing occurs, and to be better if one starts with the hard-core boson rather than the fermion.
\end{abstract}

\section{INTRODUCTION}

In two-dimensional systems there may exist (quasi)particles other than bosons or fermions. Such particles, called anyons, obey fractional statistics. ${ }^{1-3}$ A calculation of the second virial coefficient ${ }^{4}$ shows that anyon interpolates between bosons and fermions. It is believed that the quasiparticles in the fractional quantized Hall effect are actually anyons. ${ }^{5,6}$ Recently Laughlin ${ }^{7-9}$ proposed that anyons play an essential role in the high- $T_{c}$ superconductivity. After this interesting proposal, several groups have studied the free anyon system. ${ }^{8-13}$ Many of these recent studies are based on the mean-field approximation, in which the statistical flux tubes associated with the particles are replaced by a uniform magnetic field. The mean-field theory with random-phase approximation (RPA) fluctuation predicts that the ground state of the anyon system is a superfluid and becomes superconducting if the anyons are charged.

Theoretically the nature of the ground state of the free-anyon system is a fundamental problem, apart from its relation to the high- $T_{c}$ superconductivity. One of the key points is to understand the validity of the mean-field approximation. Studies to put anyons on the lattice are also performed by several authors. ${ }^{14-18}$ A pioneering computer study on a small anyon system has been done by Canright, Girvin, and Brass. ${ }^{15-17}$ They calculated the ground state of anyons on a small lattice in a cylinder geometry by exact diagonalization, and claimed that the mean-field treatment is good. They also argued, from the ground-state energy versus central-flux diagram, that there exists a paired state in some parameter range for the interparticle interaction.

We notice that a cylinder, though noncompact, is still topologically nontrivial and differs from a board or a plane in that there are noncontractible loops on it. From the path-integral formulation of fractional statistics, ${ }^{3}$ it is clear that this topological nontriviality would affect the properties of anyons through the braid group on the cylinder. And it is natural to expect that a careful reexamination based on the braid group would resolve or clarify some puzzling features in the previous results, such as the asymmetry of the ground-state energy in the magnetic flux threading through the hole of the cylinder (see Refs. 15-17).

In this paper we present an examination of anyons on a cylinder (or annulus), based on the braid-group analysis. We find that besides the usual local exchanges, there are additional generators in the braid group for a cylinder or an annulus, which correspond to moving anyons around noncontractible loops. This leads to one more parameter $\Phi$, in addition to the usual statistics parameter $\theta$, for the (one-dimensional) braid-group representations. In order to separate the effects of $\theta$ from those of $\Phi$, attention has to be paid to properly identifying the latter with the threading magnetic flux through the cylinder or annulus. This results in an unexpected necessity for modifying the usual rules for putting anyons on a square lattice when one imposes the periodic boundary condition in one direction, and we have been able to give two sets of seemingly different rules that both satisfy the braid-group requirements. Also this identification brings the following fact to light that the cylinder geometry has an extra symmetry that the annulus geometry does not have, namely, under the $180^{\circ}$ rotation in the three-dimensional space that brings either edge into the other. This symmetry requires one extra, also unexpected, restriction for anyons on a cylinder; i.e., $\exp \{2 i(N-1) \theta\}=1$, where $N$ is the total number of anyons.

To check our analysis we have performed some finite- 
system calculations. The consistency of our results has been checked by the agreement of the results from our two different sets of rules. Although they both satisfy the braid-group requirements, the equivalence between them otherwise is not obvious at all. There are some differences between our results and previous ones. ${ }^{15,17}$ For example, the fermion point in Fig. 1(a) of Ref. 17 does not agree with the exact analytic result, but our result does. Actually, one may worry that some of the previous results were based on the usual rules for putting anyons on a board, which did not incorporate the necessary modification for the cylinder or annulus that we mentioned above. In our opinion, some of the previous results correspond to systems with a certain nonzero central flux through the hole, which depends on both the particle number and the statistics. A detailed comparison will be given later.

We have also calculated the excited states and their spectral flow with either $\theta$ or $\Phi$ varying, and have seen a lot of level crossings, the existence of which seems to be a general feature of anyon systems, but the significance of the spectral flow and level crossing is not well understood yet. In particular, from our results the validity of the mean-field treatments seems to be related to level crossings. Whether starting with the fermion or the hard-core boson, the mean-field approximation looks good until the first or second level crossing occurs. Afterward the mean-field approximation is not so good. For the cylinder geometry, there are many level crossings near the fermion point but few near the hard-core boson point; and the mean-field theory seems to be much better from the latter than from the former. For comparison we also studied the mean-field approximation on the board geometry. We found it is better than on the cylinder because of less level crossings.

\section{BRAID GROUP ON A CYLINDER}

To define anyons on a cylinder (or annulus, if one temporarily ignores the differences in their geometry), we start with the braid-group analysis, which underlies the path-integral formulation of fractional statistics. ${ }^{3}$ According to the latter, the braid group is the fundamental group (or the first homotopy group) of the many-anyon configuration space, and it plays the same basic role for anyons as the permutation group for usual bosons and fermions. In particular, the scalar anyon wave function forms a one-dimensional representation of the braid group for the surface on which they are defined. So the rules describing anyons should be able to reproduce the correct phase factor for every noncontractible loop in the many-anyon configuration space in agreement with the braid-group representation. Let us first consider the continuum case in this section, then putting anyons on a lattice in the next section.

\section{A. Generators}

Recall that the braid group, $B_{N}(S)$, for $N$ anyons on a surface $S$ is the first homotopy group of the $N$-anyon configuration space:

$$
C_{N}=(S \times \cdots \times S-D) / S_{N},
$$

where because of the hard-core nature of anyons, one has to subtract the subset $D=\left\{\left(\mathbf{r}_{1}, \ldots, \mathbf{r}_{N}\right) \mid \mathbf{r}_{i}=\mathbf{r}_{j}{ }^{\exists} i \neq j\right\}$ representing configurations with at least two of the particles sitting at the same site and $S_{N}$ is the permutation group of $N$ particles which represents indistinguishability among them.

When $S$ is a plane or board, the braid group is generated by the local exchange operators:

$$
\widetilde{\sigma}_{i}, \quad i=1, \ldots, \quad N-1,
$$

where $\widetilde{\sigma}_{i}$ represent an interchange of $i$ th and $(i+1)$ th particle counterclockwise without other anyons enclosed in the exchange loop. (Note that the plane, cylinder, and annulus is all orientable and we can assign one normal direction to the surface.) These generators are known to satisfy the following relations: ${ }^{19}$

$$
\begin{aligned}
& \widetilde{\sigma}_{i} \widetilde{\sigma}_{j}=\widetilde{\sigma}_{j} \widetilde{\sigma}_{i} \quad(i \neq j \pm 1), \\
& \widetilde{\sigma}_{i} \widetilde{\sigma}_{i+1} \widetilde{\sigma}_{i}=\widetilde{\sigma}_{i+1} \widetilde{\sigma}_{i} \widetilde{\sigma}_{i+1} .
\end{aligned}
$$

The braid group of the cylinder is more complicated, since the first homotopy group of the cylinder is nontrivial: Namely, there are noncontractible loops even for a single particle on the cylinder, and this must affect the first homotopy group for the many-particle configuration space. More concretely, for a cylindrical system, there is another type of generator $\widetilde{\rho}_{j}$, which represents moving the $j$ th particle (e.g., $j$ th from the left or inner edge of the cylinder or annulus) around the hole once in the positive direction, with $j-1$ particles between it and the left or inner edge (see Figs. 1 and 2), similar to those on a torus. $^{20}$ It is easy to see that these generators $\widetilde{\rho}_{j}$ $(j=1,2, \ldots, N)$ satisfy

$$
\begin{aligned}
& \widetilde{\rho}_{i} \widetilde{\rho}_{j}=\widetilde{\rho}_{j} \widetilde{\rho}_{i} \quad(i \neq j), \\
& \widetilde{\sigma}_{i} \widetilde{\rho}_{j}=\widetilde{\rho}_{j} \widetilde{\sigma}_{i} \quad(i \neq j, i+1 \neq j), \\
& \widetilde{\rho}_{j+1}=\widetilde{\sigma}_{j} \widetilde{\rho}_{j} \widetilde{\sigma}_{j},
\end{aligned}
$$

where (2.5) and (2.6) are clear and (2.7) can be understood from Fig. 1. We emphasize that the existence of the additional braid-group generators $\widetilde{\rho}_{j}$ is a consequence of the existence of noncontractible loops on the cylinder.

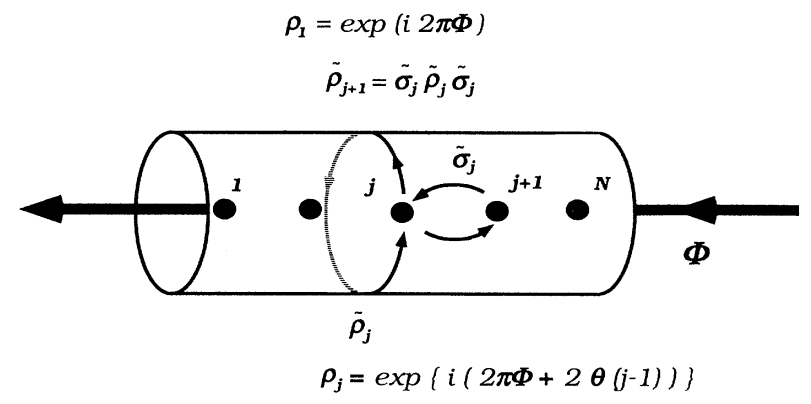

FIG. 1. The cylinder with generators for the braid group on it. $\Phi$ is a central flux which is through the hole of the cylinder. 

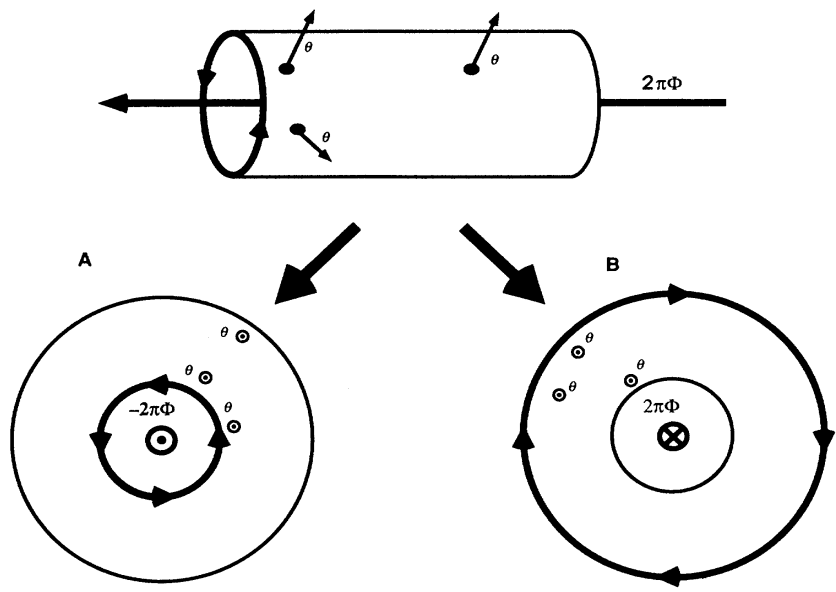

FIG. 2. Two different methods to deform a cylinder into annuli. The two annuli $A$ and $B$ are different as an annulus. They should be the same, however, if we consider the system as a cylinder.

\section{B. Representations}

Now let us examine the one-dimensional unitary representations of the braid group. For $\widetilde{\sigma}_{j}$ we get the representation $\sigma_{j}=e^{i \theta}$ (for all $j$ ) as usual and we can interpret $\theta$ as the anyon statistics. ${ }^{3}$ Then (2.6) implies that the representation for $\widetilde{\rho}_{j}$ satisfies

$$
\rho_{j+1}=\rho_{j} \exp [i 2 \theta] .
$$

Using (2.8) repeatedly we obtain

$$
\rho_{j}=\rho_{1} \exp [i 2 \theta(j-1)] \text {. }
$$

We note that because of $(2.8)$, different $\widetilde{\rho}_{j}$ are represented by different phases; though at first glance it is somewhat counter intuitive for the cylindrical case, but upon further reflection one may convince oneself that this is correct.

Now we come to one main point of this paper. To fix the braid-group representation we need to fix the phase of $\rho_{1}$. This implies that the existence of the generators $\widetilde{\rho}_{j}$ requires an additional parameter, in addition to $\theta$, to label the braid-group representations on a cylinder or annulus. Physically this is not very surprising, since the existence of the hole makes it necessary to consider the Aharonov-Bohm effect, that is, the effects of a central flux $\Phi$ threading through the hole (see Figs. 1 and 2). Naturally we include the effects of this flux by setting

$$
\rho_{1}=\exp [i 2 \pi \Phi] \text {. }
$$

In other words we define physical flux $\Phi$ by this equation. Thus $\rho_{j}$ is given by

$$
\rho_{j}=\exp \{i[2 \theta(j-1)+2 \pi \Phi]\} .
$$

$\dot{W}$ e see that the two parameters $\theta$ and $\Phi$ are tangled in the phase of $\rho_{j}$. This is an unavoidable consequence of the topological nontriviality we mentioned several times. And it turns out to be important later for correct rules for putting anyons on a lattice, where one needs to properly entangle the two parameters. Also we emphasize that (2.10) or (2.11) is a natural and physical definition of the flux $\Phi$ through the hole since, by our definition, $\widetilde{\rho}_{1}$ represents moving an anyon around the inner edge of the annulus or the left edge of the cylinder without enclosing other anyons.

\section{Symmetry and restriction}

The above consideration immediately leads to the following consideration: Unlike the annular case, the two edges of a cylinder are symmetric; one should be able to identify the phase for the generator $\widetilde{\rho}_{N}$ at the right edge with the central flux $\Phi$ as well. Using (2.11) with $j=N$ one immediately obtains the constraint

$$
\exp [i 2 \theta(N-1)]=1 \text {. }
$$

With $N$ given, the allowed values of $\theta$ are restricted to be

$$
\theta=\frac{m \pi}{N-1} \quad(m=0, \ldots, 2 N-3) .
$$

If $\theta / \pi$ is fixed to be fractional, then the total number $N$ of the anyons cannot be arbitrary.

Putting it in another way, let us rotate the cylinder in Fig. 3(A) in three dimensions by $180^{\circ}$ as shown in Fig. 3(B). This symmetry operation exchanges the two edges
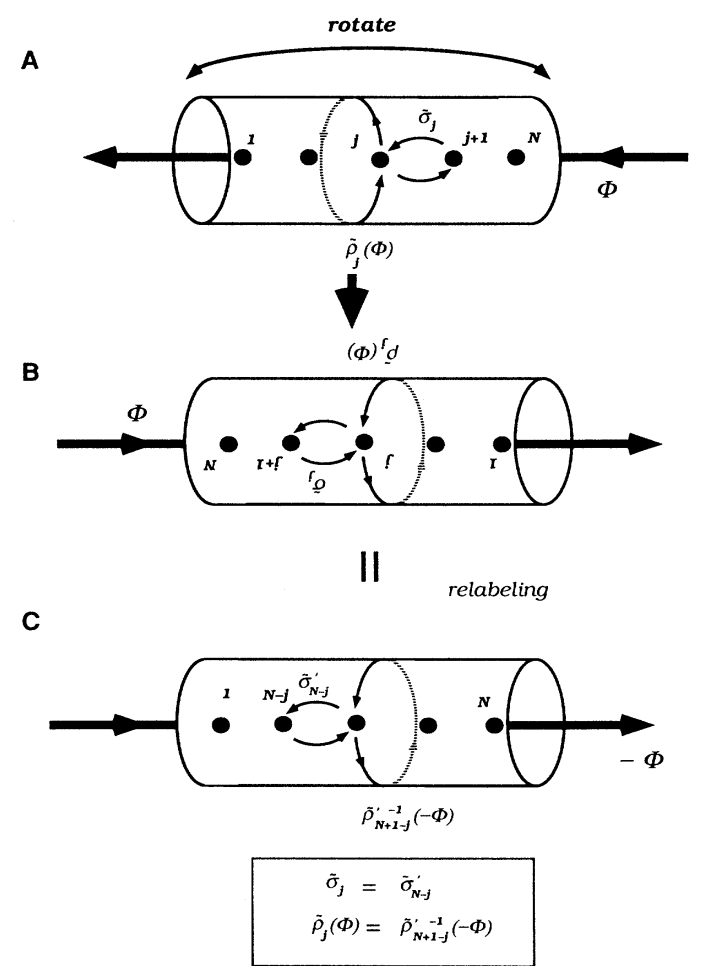

FIG. 3. Schematic figure to explain the restriction for the statistics on the cylinder. The cylinder has an extra symmetry that the annulus does not have, namely, under the $180^{\circ}$ rotation in the three-dimensional space that brings either edge into the other. 
and reverses the direction of the central flux. However, the direction of the statistical flux of the particle does not change. Now let us relabel the particles in Fig. 3(C) and denote the new generators with prime like $\widetilde{\sigma}^{\prime}$ :

$$
\begin{aligned}
& \widetilde{\sigma}_{j}=\widetilde{\sigma}_{N+1-j}^{\prime}, \\
& \widetilde{\rho}_{j}(\Phi)=\widetilde{\rho}_{N+1-j}^{-1}(-\Phi),
\end{aligned}
$$

where the inverse on the right-hand side of (2.15) is present because the direction of $\tilde{\rho}_{j}$ is reversed by the rotation procedure. Because of the symmetry of the cylinder, this relabeling should not change any physics and, therefore, the new generators should be represented in the same way. Thus (2.15) gives

$$
\begin{aligned}
& \exp [i(2 \theta(j-1)+2 \pi \Phi)] \\
& \quad=\exp [-i\{2 \theta(N+1-j-1)-2 \pi \Phi\}] .
\end{aligned}
$$

This leads to the same constraint (2.12).

A third argument can be given for the restriction (2.12) as follows: We let one anyon go around all other anyons counterclockwise which produces a phase $e^{i 2(N-1) \theta}$. Now let us deform the loop on the cylinder as shown in Fig. 4. We obtain a loop consisting of two loops at the opposite edges with opposite orientation and a line from one edge to the other and back forth. Since here we only consider Abelian phases, the contributions from the latter line cancel during traveling back and the contributions from the two edges cancel against each other because of the symmetry between the edges of the cylinder and their opposite orientation. Thus, though the above loop is noncontractible, it produces a trivial phase factor of unit on a cylinder. Again this gives rise to (2.12), which is similar to the restriction on a sphere. ${ }^{21}$

We note that physically (2.15) implies that on the cylinder, reversing the direction of the central flux $\Phi$ will not change the spectrum of the anyon system. As a consequence, one infers that changing only the sign of $\theta$
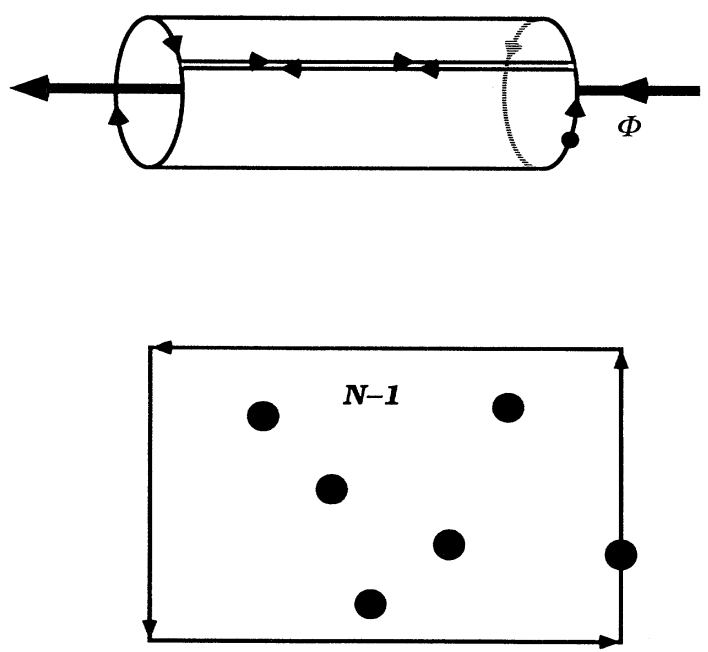

FIG. 4. Schematic figure to explain the restriction for the statistics on the cylinder. will not change the spectrum either. (This is because changing the sign of both $\theta$ and $\Phi$ only turns the representation into its complex conjugate.) These are certainly desired symmetries for the cylinder case, since the central flux is perpendicular to the statistical flux. In this way we see that to recover these symmetries anyon statistics or the number of anyons has to be constrained by (2.12). The anyons on annulus does not necessarily satisfy this restriction. This tells us that though the cylinder and annulus are topologically equivalent, however, the special geometric symmetry of the cylinder has effects on anyons on it. This is also an evidence showing that compared with the planar case, the nontrivial topology of the cylinder can have observable effects on physics of anyons in a finite system. (As in the spherical case, these topology-dependent effects are expected to disappear in the thermodynamic limit, since when $N$ tends to infinity, the allowed values of $\theta$ becomes quasicontinuous.)

To conclude this section let us comment on symmetries of anyons on an annulus. First we note than an annular anyon system described by the representation $(\theta, \Phi)$ should have the same spectrum as that by $(-\theta,-\Phi)$, since the two systems are related to each other by reversing the orientation of the annulus. Generically it is expected that the $\pm \theta$ anyons on an annulus with the same nonzero central flux do not have the same spectrum, since their statistical fluxes have opposite direction with respect to that of $\Phi$. However, when the value of $\theta$ satisfies (2.12), one can establish relations like (2.14)-(2.16) for two identical annular systems with the central flux reversed, which imply that such an annular $(\theta, \Phi)$ anyon system does have the same spectrum as that for $(\theta,-\Phi)$ and for $(-\theta, \Phi)$. Later we will perform numerical calculations to check these symmetries.

\section{PUTTING ANYONS ON A LATTICE}

To investigate anyons numerically one has to be able to define anyons on a two-dimensional lattice with appropriate boundary conditions. The guiding principle for doing this is to produce the phase factors for all noncontractible loops in the many-body configuration space which are consistent with the braid-group representation. In particular, the braid-group relations for its generators have to be respected. In the cylinder or annulus case, attention has to be paid to the relations and values for the additional generators corresponding to moving anyons around the hole.

In this section we first review the (square) lattice Hamiltonian and anyons on a board, then discuss the necessary modifications for the cylinder or annulus. By "board" we mean the vanishing boundary condition for both $x$ and $y$ directions; for a "cylinder" or "annulus" one has the vanishing boundary condition for the $x$ direction, but the periodic boundary condition for the $y$ direction.

\section{A. General Hamiltonian}

If two anyons are interchanged, the total wave function of the system changes by a phase factor $e^{i \theta}$. First one has to assume that the particles have a hard core, which is 
crucial for the existence of anyons. ${ }^{1,3}$ Then one can imagine an anyon as a particle having a unit charge and a flux tube of strength $\theta$, and interpret the exchange-phase $e^{i \theta}$ as due to the Aharonov-Bohm phase associated with the flux tube. ${ }^{2,3}$ When two particles are interchanged, each particle feels the other's flux so that the total phase change is $e^{i \theta}$. This argument is important for a meanfield treatment.

We represent the flux tube adjoined to an anyon by drawing a string from the dual site to the boundary and assign a phase $e^{i \theta}$ to each of the links cut by the string, or more precisely to each hopping matrix element on such a link. Thus, a lattice anyon is defined as a particle with an associated string, which has only one end point and goes to the infinity or the boundary (see Fig. 5). The Hamiltonian of the system is given by

$$
H=-\sum_{\langle i j\rangle} t_{i j} c_{j}^{\dagger} e^{i \theta i j} c_{i}+\text { H.c. , }
$$

where the summation is over the nearest neighbors and $c_{i}$ is the hard-core boson operator at site $i,{ }^{14}$ satisfying

$$
\begin{aligned}
& {\left[c_{i}^{\dagger}, c_{j}\right]=\delta_{i j},} \\
& c_{i}^{\dagger} c_{i}^{\dagger}=0 .
\end{aligned}
$$

The phase factor $\theta_{i j}=-\theta_{j i}$ is defined on the link $\langle i j\rangle$. If we include a real magnetic field, then $\theta_{i j}$ is written as

$$
\theta_{i j}=\sum_{\text {string } \in \operatorname{link}\langle i j\rangle} \pm \theta+2 \pi \Phi_{i j},
$$

where $\Phi_{i j}$ is a contribution from the real magnetic field. Here we identify $\theta_{i j}$ as $(2 \pi e / c h) \int_{i}^{j} \mathbf{A} \cdot d \mathbf{l}$, where $\mathbf{A}$ is a total vector potential of the statistical flux and the real magnetic field; the quantity

$$
\frac{1}{2 \pi} \sum_{\text {around } S} \theta_{i j}=\frac{e}{c h} \oint_{\partial S} \mathbf{A} \cdot d \mathbf{l}=\frac{1}{\phi_{0}} \int_{S} \mathbf{B} \cdot d \mathbf{S}
$$

is the total (statistical plus real) magnetic flux through the area $S$ in units of the flux quantum $\phi_{0}=c h / e$. The sign of the statistics part of $\theta_{i j}$ depends on the direction of hopping across the string. The Hilbert space of the system is spanned by the basis which specifies the position of the particles,

$$
\left|\left\{\mathbf{r}_{1}, \mathbf{r}_{2} \ldots, \mathbf{r}_{N}\right\}\right\rangle,
$$

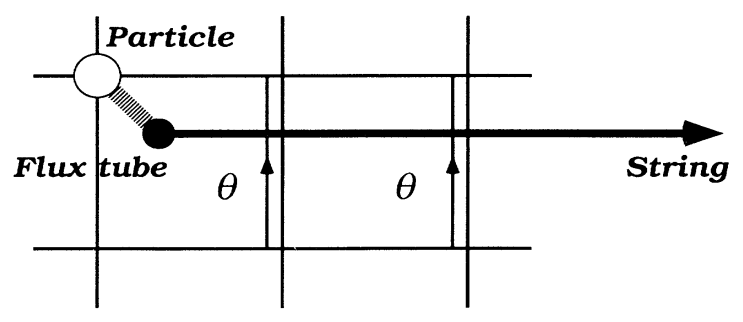

FIG. 5. The configuration of a particle and a flux. The white circle is the particle and the black circle is the flux tube. The line with arrow is a string to represent the flux tubes. If the string cuts links, we give a phase factor $e^{i \theta}$ to the hopping matrix element across the link. where $\mathbf{r}_{j}$ is the two-dimensional coordinate of the $j$ th particle. Here the order of $\mathbf{r}_{j}$ is irrelevant in this basis. From (3.1) and (3.4) we can determine the hopping matrix elements between the base vectors (3.5).

We have not specified the boundary condition yet. The key point here is that the choice of the set of phase factors $\theta_{i j}$, or the choice of anyon strings, has to be compatible with the boundary conditions in the sense of agreeing with the braid-group requirements. Now let us consider this problem step by step.

\section{B. Rule A (for a board)}

By the argument of the previous section it is easy to define the anyons on a board. We consider an $L_{x} \times L_{y}$ square lattice and define the anyon system by assigning strings as shown in Fig. 6. Every anyon string is parallel to the $x$ direction and runs from left to right all the way to the boundary. If another anyon hops from down to up across such a horizontal string, the change of the phase is given by $e^{+i \theta}$; the hopping in the opposite direction across the string would give rise to the opposite phase. When a particle moves, the string attached to it moves accordingly. The phase change caused by the sweep of the moving string over other anyons is determined as if those anyons hop across the string.

We emphasize that since anyons are identical particles, all the anyon strings have to be the same. One cannot simply have some of the anyon strings running in the opposite direction, since it is easy to see that if so, not every exchange of a pair of anyons (without enclosing other particles) will give the same phase $e^{i \theta}$, though moving one anyon around the other once always gives the right phase $e^{i 2 \theta}$.

There is a subtlety involving the configurations in which more than one particle has the same $y$ coordinates. When one of them hops in the $y$ direction, we have to give a rule to determine unambiguously how many strings it goes across and how many other anyons are swept by the string of the hopping anyon, so that exchanging a pair of such anyons should lead to the same

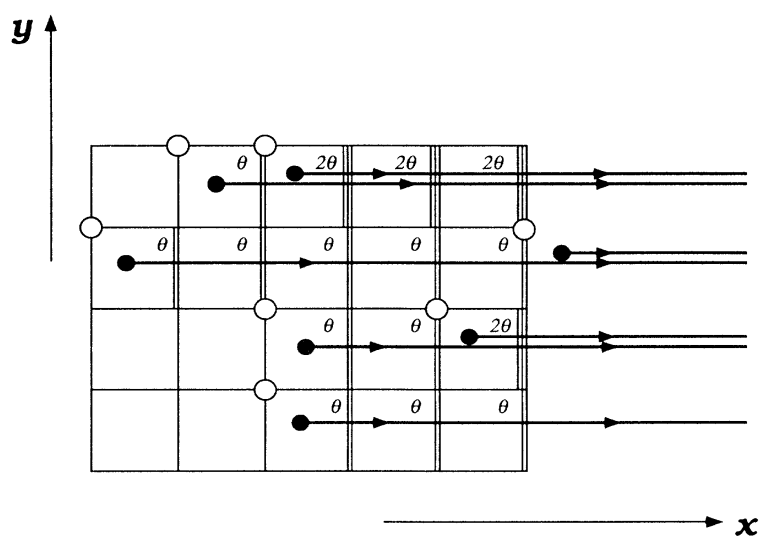

FIG. 6. The string rule for the board geometry. The white circles are the positions of particles and the black circles are the positions of flux tubes. We call this string rule as rule $\mathrm{A}$. 
phase $e^{i \theta}$. To achieve this we shift the position of the flux tubes from $\left(m+\frac{1}{2}, n-\frac{1}{2}\right)$ to $\left(m+\frac{1}{2}, n-\frac{1}{2}+m \delta\right)$, where $\delta$ is a infinitesimally small positive number, so that for anyons with the same $y$ coordinate, the particles more to the left have a lower string.

With all these specifications, now one can verify that all exchange phases associated with $\widetilde{\sigma}_{i}$ 's are in agreement with (2.3) and (2.4). We call the above rules as rule A, which consistently describes the anyons on a board. However, in the next subsection, we will see that without modification it does not apply to the cases when the periodic boundary condition is imposed on one direction.

\section{Rule B (for an annulus or cylinder)}

Let us impose the periodic boundary condition in the $y$ direction of the lattice. We have to model the anyons on such a lattice to satisfy the braid-group representation relations on a cylinder or annulus that we discussed in the previous section.

The relations for the $\sigma_{j}$ 's are satisfied by rule A, i.e., the assignment of anyon strings as in Fig. 6. But as emphasized before, we have to take into account the generators $\rho_{j}$ 's, i.e., the phase $[2 \theta(j-1)+2 \pi \Phi]$ for the $j$ th anyon moving around the hole. The $2 \pi \Phi$ part can be easily included into the phase of each hopping amplitude along a link in the $y$ direction by changing it from real $t$ to $t \exp \left[i 2 \pi \Phi / L_{y}\right]$. Thus, whenever an anyon moves around the hole in the positive direction, an extra phase $2 \pi \Phi$ is added to that obtained by rule $\mathrm{A}$.

Let us check whether these rules can give correct phases in consistency with the braid-group relations (2.8) and (2.10). To do this we need to carefully consider the hopping in the periodic direction (i.e., $y$ direction) around the hole. If no two anyons have the same $x$ coordinate, then it is easy to verify that the relation (2.8) is always satisfied. Since for the $i$ th particle from the left edge, there are $i-1$ particles on its left and $N-i-1$ on its right, contributing opposite phases to $\rho_{i}$. So $\rho_{i+1}$ differs from $\rho_{i}$ by a factor $e^{i 2 \theta}$. However, when one evaluates the phase of, say, $\rho_{1}$, one immediately realizes that it does not satisfy the relation (2.10). The phase $\rho_{1}$ for the leftmost anyon moving in the $y$ direction, obtained from rule A supplemented by that for $\Phi$, is not the right one: it is $-(N-1) \theta+2 \pi \Phi$ but not $2 \pi \Phi$, since in implementing $\rho_{1}$ the moving anyon string sweeps over all $N-1$ anyons to its right. This is the problem we emphasized before: The effects of $\theta$ and $\Phi$ are tangled in $\rho_{i}$. So rule A is actually wrong in having a wrong value for the central flux by an amount of $-(N-1) \theta$. To correct this we simply add a cut or string at $y=\frac{1}{2}$ from the left edge to the right edge and let it carry the strength or phase jump $(N-1) \theta$, so that every anyon going across it upward will gain an extra phase $(N-1) \theta$, compensating the error brought by simply applying rule $A$ on an annulus. With this rule supplemented to those of rule $A$, we obtain rule $B$, which correctly describes the anyons on an annulus by having properly isolated the statistics $\theta$ from the central flux $\Phi$. For the cylinder case we simply require, in addition, that $N$ and $\theta$ satisfy the restriction (2.12).

We notice that our correction is formally equivalent to shift the central flux, since the braid-group relation (2.10) gives an unambiguous definition for the central flux $\Phi$. Alternatively the above correction of the central flux can be achieved by changing the hopping amplitude along each $y$ link to $t \exp \left\{i[2 \pi \Phi+(N-1) \theta] / L_{y}\right\}$. Then one does not need the cut at $y=\frac{1}{2}$ and this treatment will explicitly maintain periodicity everywhere.

One may think that the situations with more than one particle having the same $x$ coordinate are more delicate. However it is not much so, since in implementing $\rho_{i}$ for one such particle, horizontal hopping has to occur somewhere to avoid the anyons standing on its way in the $y$ direction, and not any ambiguity would arise consequently. Thus, rule B is enough to deal with all situations.

Another more intuitive derivation of rule $\mathbf{B}$ is given in the Appendix without explicitly involving the braidgroup arguments.

As a consistency check let us show that for the $\theta=\pi$ case, rule $B$ reproduces the usual rule for the Fock space representation of the fermionic Hilbert space. The Hilbert space is spanned by

$$
f_{i(1)}^{\dagger} f_{i(2)}^{\dagger} \ldots f_{i(N)}^{\dagger}|0\rangle
$$

where $f_{i(j)}^{\dagger}$ is a creation operator of the usual fermion at site $i(j)$ and the sites $i(j)$ 's are ordered by some convention. An ordering convention which is consistent with rule $B$ is

$$
(1,1)<(2,1)<(3,1)<\cdots<\left(L_{x}, 1\right)<(1,2)<(2,2)<\cdots<\left(L_{x}, L_{y}\right) .
$$

If the hopping particle is not across the periodic boundary, e.g., from $(m, n)$ to $(m, n+1)\left(n<L_{y}\right)$, (3.7) tells us the sign of the hopping process is given by

$$
(-1)^{L+R} \text {, }
$$

where $L$ is the number of the particles whose coordinate is $(x, n+1)(x \leq m-1)$ and $R$ is the number of the particles whose coordinate is $(x, n)(x \geq m+1)$. By rule $\mathrm{A}$ or rule $B$, the change of the phase is given by

$$
\exp [i(L-R) \pi]=(-1)^{L-R}
$$

It coincides with the fermion sign. However, if the fermion at point $P$ in Fig. 7(a) hops to point $Q$, the ordering convention tells us the sign is

$$
(-1)^{\mathcal{N}(A)+\mathcal{N}(D)+\mathcal{N}(E)}
$$

where $\mathcal{N}(X)$ is the number of particles in region $X$. By rule $B$, the change of the phase is given by 
(a)

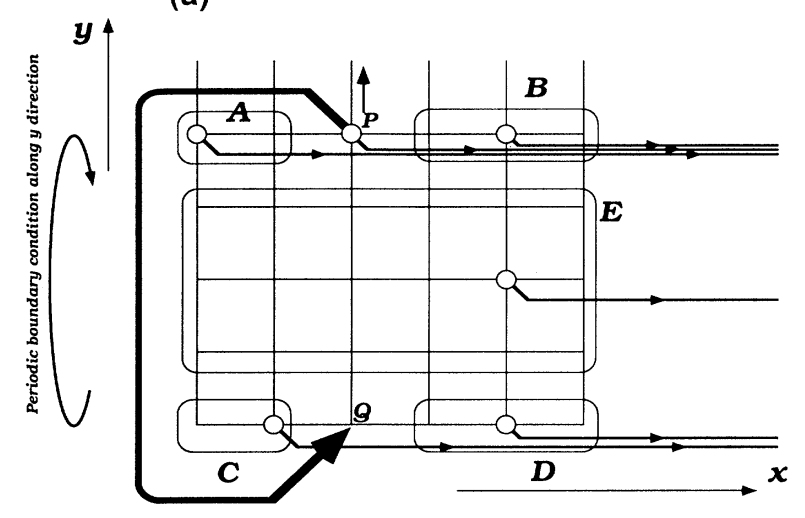

(b)

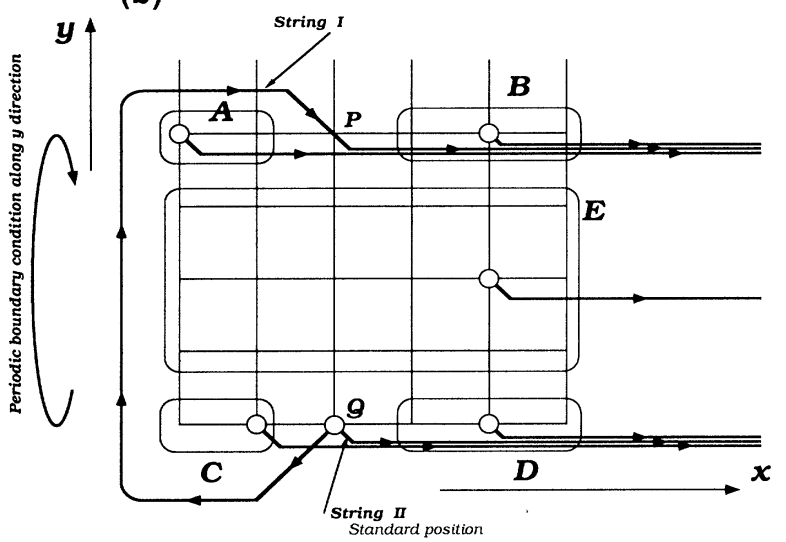

FIG. 7. (a) A hopping process in which a particle hops at the edge of the system under the periodic boundary condition. (b) The string configuration after the hopping. These figures explain rule B. String I shows the configuration just after the hops. String II is the standard position of the string.

$$
\begin{aligned}
\exp [i\{(N-1)+\mathcal{N}(C)-\mathcal{N}(B)\} \pi] & \\
& =(-1)^{\mathcal{N}(A)+\mathcal{N}(D)+\mathcal{N}(E)},
\end{aligned}
$$

in agreement with (3.10), where $\mathcal{N}(A)+\mathcal{N}(B)+\mathcal{N}(C)$ $+\mathcal{N}(D)+\mathcal{N}(E)=N-1$ is used.

However, if we use rule $\mathrm{A}$, this phase is given as $(-1)^{\mathcal{N}(C)-\mathcal{N}(B)}$, in conflict with the fermion rule (3.10). So we conclude that rule $\mathrm{A}$, when naively applied to the annulus or the cylinder, cannot even reproduce the free fermions by starting from the hard-core bosons. Indeed, we have noticed that in Fig. 1(a) of Ref. 17 for the ground-state energy versus statistics, the fermion point does not agree with the exact result from the usual fermion calculation. It is expected that the whole curve there is not correct, since it actually corresponds to the cases with a nonvanishing central flux $-(N-1) \theta$, which depends on $\theta$.

\section{Rule C (also for an annulus or cylinder)}

There is another rule which can consistently define the anyons on the annulus or cylinder. Of course, $\Phi$ should

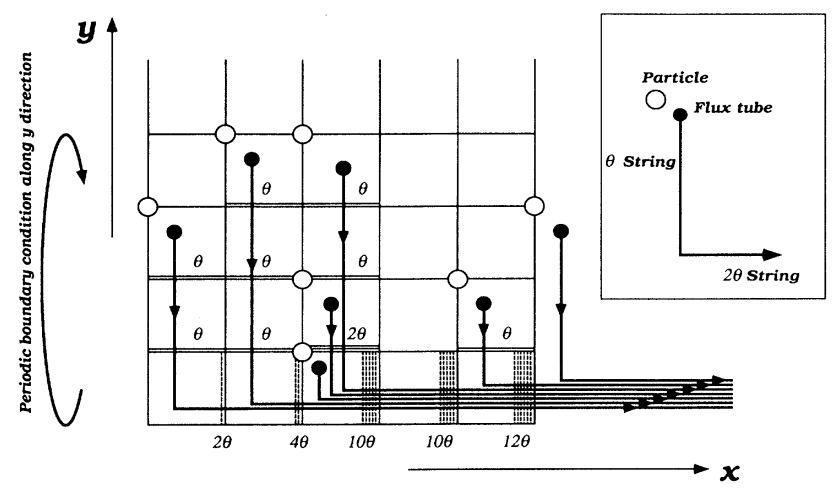

FIG. 8. Another string rule for the cylinder. We call this string rule as rule $\mathrm{C}$.

be included as usual. We call the following rule as rule $\mathrm{C}$ : Assign to each anyon located at, say, the site $(m, n)$ a $\theta$ string from the adjoining plaquette $\left(m+\frac{1}{2}+n \delta, n-\frac{1}{2}\right)$ vertically to $\left(m+\frac{1}{2}+n \delta, \frac{1}{2}\right)$ where it is turned into a horizontal $2 \theta$ string to the right edge, as shown in Figs. 8 and 9 . Here $\delta$ is an infinitely small positive number to avoid the ambiguity in the positions of the vertical strings when several particles have the same $x$ coordinate. This rule is similar to that given in Ref. 18 for anyons on a torus.

For various noncontractible loops in the anyon configuration space, one can straightforwardly verify the consistency of rule $\mathrm{C}$ for the anyon string with the braidgroup relations on the annulus or cylinder. Especially the horizontal $2 \theta$ strings are designed for the relation (2.8) to hold, with $\rho_{i}$ realized as a closed hopping along the $y$ direction across $i-1$ such strings. Although the cut at $y=\frac{1}{2}$ appears to have broken the periodicity in the $y$ direction, no real breaking occurs. But the advantage of this rule is that no correction like in rule $\mathbf{B}$ for the central flux $\Phi$ is needed, since it is easy to see that for the leftmost anyon, $\rho_{1}$ is exactly $\exp \{i 2 \pi \Phi\}$.

As a check, we show that rule $\mathrm{C}$ is also consistent with the usual fermion rule if we take the ordering convention as

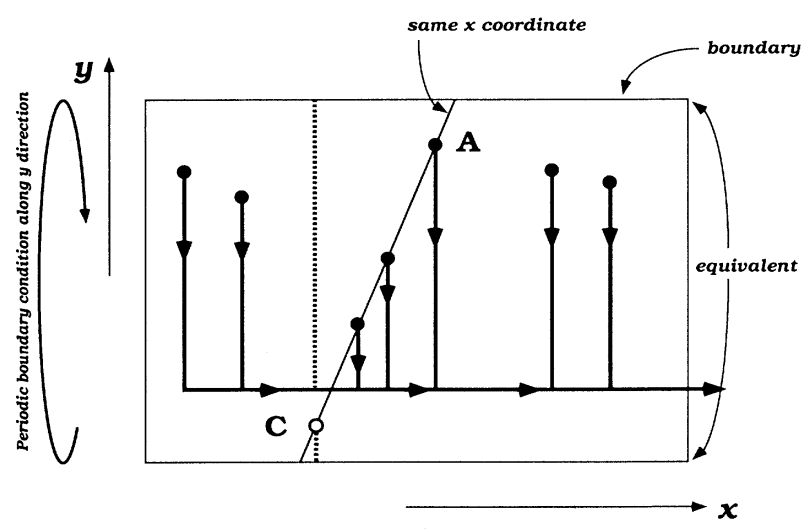

FIG. 9. A schematic figure to express the delicate phase in rule $C$. The black circles express the composite of a particle and a flux tube. 


$$
(1,1)<(1,2)<(1,3)<\cdots<\left(1, L_{y}\right)<(2,1)<(2,2)<\cdots<\left(L_{x}, L_{y}\right) .
$$

For the cylinder case, there are some subtle points. Consider the case in which the particle at the site $\left(m, L_{y}\right)(A$ in Figs. 9 and 10) hops to the site $(m, 1)(C$ in Figs. 9 and 10). The usual fermion rule tells us that the phase should be $(-1)^{M_{m}-1}$, where $M_{m}$ is the number of particles which have the same $x$-coordinate $m$ (in Fig. 9, $M_{m}=3$ ). To take into account this phase we divide the hopping process into two parts. First, the particle at $A$ hops to a virtual $B$ site [Fig. 10(a)]. There is no phase change in the process. Next it hops from the site $B$ to $C$ [Fig. 10(b)]. The phase change in this process is

$$
\exp \left[i \theta\left(M_{m}-1\right)\right]
$$

because the $M_{m}-1$ particles are swept by the $\theta$ string of the moving particle in the positive direction. For a fermion, $\theta$ is equal to $\pi$, thus $(3.13)$ is $(-1)^{M_{m}-1}$, which is consistent to the usual fermion rule.

Let us proceed to check whether rule $\mathrm{C}$ correctly represents the generator $\rho$ or not. We continue the process until the particle returns to the original point $A$. If the particle hops from $C$ to $D$ [Fig. 10(c)], the change of the phase is $\exp \left[i 2 \theta N_{m}\right]$, where $N_{m}$ is the number of the particles whose $x$ coordinates are less than $m$. We assume that the string loop disappears completely after the particle crosses the line $y=\frac{1}{2}$ where the $2 \theta$ strings are lying. Next it hops from $D$ back to $A$ and the phase change is $\exp \left[-i \theta\left(M_{m}-1\right)\right]$. Then the total phase change in the process in which the particle at $A$ moves around the cylinder by the route shown in Fig. 10(d) is

$$
\exp \left[i \theta\left(M_{m}-1\right)\right] \exp \left[i 2 \theta N_{m}\right] \exp \left[-i \theta\left(M_{m}-1\right)\right]=\exp \left[i 2 \theta N_{m}\right]
$$

which is $\rho_{N_{m}+1}$ as expected.

Rule $\mathrm{C}$ for anyons on the annulus or the cylinder looks very different from the previous rule $B$. The equivalence between them can be established indirectly by showing that for each generator $\widetilde{\sigma}_{j}$ and $\widetilde{\rho}_{j}$, the two rules always give identical phases. As we will show later, numerical calculations also verifies the equivalence. We emphasize that rule $\mathrm{C}$ looks very natural in dealing with the central flux, and so its equivalence with rule B confirms that our correction for the central flux in the latter is really correct.

We conclude this section by making the following remark. A lattice has no geometry. Our lattice described by rule $\mathrm{B}$ or rule $\mathrm{C}$ generically corresponds to an annulus system. But when the condition (2.12) is satisfied, it

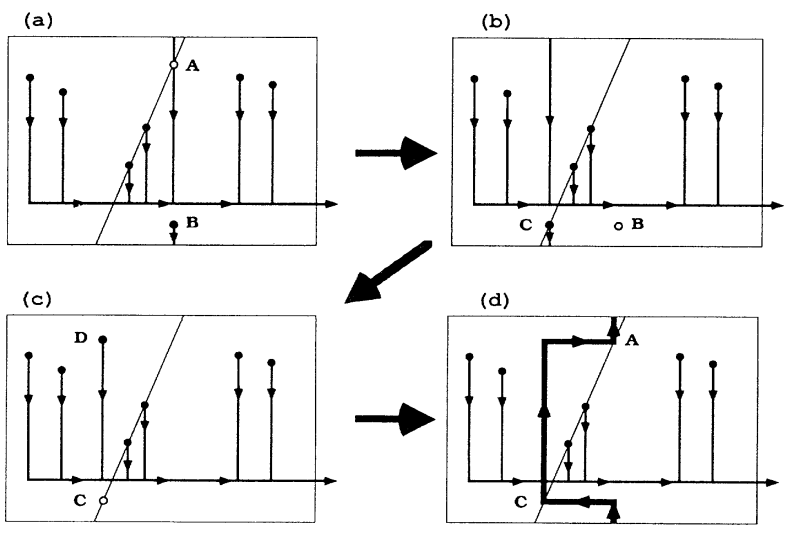

FIG. 10. Schematic figure to count the phase in the process in which a particle hops from $A$ to $C, C$ to $D$, and D to $A$ by rule $\mathrm{C}$. represents either a cylindrical system or one of two identical annular systems with opposite $\Phi$ (see Fig. 2). This agrees with the known equivalence of the latter two free systems satisfying (2.12) in the continuum case, since the braid-group relations (2.3)-(2.11) hold unchanged during the continuous deformations shown in Figs. 2(A) and 2(B), which change the cylinder into the two annuli with opposite $\Phi$.

\section{NUMERICAL RESULTS}

We treat an $L_{x} \times L_{y}$ finite-size system with $N$ particles by an exact diagonalization and we denote it by $L_{x} \times L_{y} / N$. For a small system, we use the usual packages of a combination of the Hausholder method (to get eigenvalues) and the bisection method (to get eigenvectors). We can get all the states for a small system such as $3 \times 3 / 3$. For a larger lattice we also use the Lanczos method to obtain eigenenergies.

\section{A. Consistency with the analytical argument}

We want to check the consistency of our numerical calculation with the analytical argument presented in the last section. First we compare the energy of $2 \pi \frac{1}{2}$ anyons to that of usual fermions (tight-binding model). Our results are completely consistent with it both for the board and the cylinder geometries. One point we have to note in the cylinder geometry is the following. We have constructed two sets of rules to satisfy braid-group arguments, rules $B$ and $C$. We believe that they are equivalent. However, it is not so simple to show the equivalence directly. We investigate the consistency by the numerical calculation of the ground-state energies. We have calculated the total energies for a $3 \times 4 / 6$ annulus geometry by these two rules and rule $\mathrm{A}$. The re- 
sults are shown in Fig. 11. Rules B and C give completely the same results for general statistics on the annulus geometry. If we use rule $\mathrm{A}$, the energies are different. We cannot reproduce the free fermion value by rule $A$ (here $-6-2 \sqrt{2}$ shown in Fig. 11 indicates the exact free fermion energy). It seems that Fig. 1 of Ref. 15 and Fig. 1(a) of Ref. 17 coincide with those calculated by rule $A$.

Next we want to investigate the effects of the central flux which threads through the hole of the annulus. Results for a $3 \times 3 / 4$ system with various statistics parameters are shown in Figs. 12(a) -12 (c), results for a $3 \times 4 / 6$ system are shown in Fig. 12(d) and results for a $3 \times 3 / 3$ are shown in Fig. 12(e). For each case we calculate ground-state energies of $\pm \theta$ anyons versus $\Phi$, where $\theta / 2 \pi$ 's are $\frac{1}{4}$ (semion), $\frac{1}{5}$ and $\frac{1}{6}$ for the $3 \times 3 / 4$ system, $\frac{1}{10}$ for the $3 \times 4 / 6$ system, and $\frac{1}{4}$ (semion) for the $3 \times 3 / 3$ system. In general, we have $E(\theta, \Phi) \neq E(-\theta, \Phi)$ and $E(\theta, \Phi) \neq E(\theta,-\Phi)$. So it is not surprising that on an annulus the energy is not symmetric with respect to reversing $\Phi$. However, if the condition $(2.12)\left(e^{i 2 \theta(N-1)}=1\right)$ is satisfied, that is $\theta / 2 \pi= \pm \frac{1}{6}$ for $3 \times 3 / 4, \theta / 2 \pi= \pm \frac{1}{10}$ for $3 \times 4 / 6$, and $\theta / 2 \pi= \pm \frac{1}{4}$ (semion) for $3 \times 3 / 3, \pm \theta$ anyon's energies are exactly the same. If the system is a cylinder, we cannot distinguish $\pm \theta$ statistics and the equation $E(\theta, \Phi)=E(-\theta, \Phi)$ holds. Also the energy is invariant if we change the signs of $\theta$ and $\Phi$ simultaneously. This implies that $E(\theta, \Phi)=E(\theta,-\Phi)$ and there is no asymmetry with respect to $\Phi$ in the energy diagram for the cylinder system [see Figs. 12(c), 12(d), and 12(e)].

We have also calculated an $E-\Phi$ diagram by introducing a next-nearest-neighbor interaction $u$. We calculated for the case $u=-0.5$ which corresponds to the cases in Fig. 2 of Ref. 16. The results obtained from our rule agree with theirs. It seems our rule and their rule in Ref. 16 are consistent. This means the rules used in Refs. 15 and 16 are different.

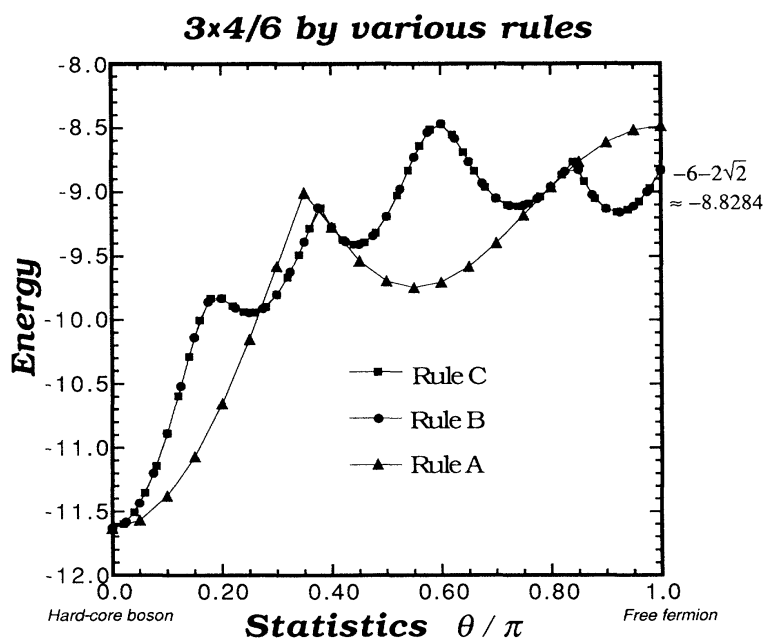

FIG. 11. Ground-state energy vs statistics diagram by three different rules for a $3 \times 4 / 6$ annulus system.

\section{B. Spectral flow and level crossing}

We calculated not only the ground-state energies, but also excited energies. We find that there are many level crossings when we sweep the central flux. The Hamiltonian of the system is invariant if we change $\Phi$ by an integer. A change of $\Phi$ by $\Delta \Phi$ is removed by a redefinition of the original hard-core-boson operators (3.2) as

$$
c_{m, n} \rightarrow c_{m, n} \exp \left(-i 2 \pi \Delta \Phi \frac{n}{L_{y}}\right),
$$

where $c_{m, n}$ is the annihilation operator of the hard-core boson at $(m, n)$ site. If $\Delta \Phi$ is an integer, (4.1) does not cause any effect to the boundary condition, that is, $c_{m, L_{y}+1}=c_{m, 1}$ is also satisfied for the new operators. We calculated the lowest several energies for various systems. In Fig. 13 we show the results (a) for $2 \pi \frac{1}{5}$ statistics in the $3 \times 3 / 4$ system, (b) for $2 \pi \frac{1}{10}$ statistics in the $3 \times 4 / 6$ system, (c) for $2 \pi \frac{1}{10}$ statistics in the $3 \times 3 / 3$ system, and (d) for $2 \pi \frac{1}{4}$ statistics (semion) in the $3 \times 3 / 3$ system. These show that the spectrum is really invariant if we change $\Phi$ to $\Phi+1$, however, the period of each level is not one. This is the so-called spectral flow. In Fig. 13(a), the lowest three levels are separated from the other levels (there is an energy gap) and the period of the states is three. We can explain this by a simple consideration. We can remove the effect of the change from $\Phi$ to $\Phi+1$ by a gauge transformation (4.1) and we can interpret this gauge transformation as a "large" gauge transformation because this gauge transformation is not continuously connected to the identity. On the other hand if the change of the central flux is $L_{y},(4.1)$ becomes an identity. Thus the period of the whole system has to be $L_{y}$. It is also possible that the period is not $L_{y}$ but a factor of $L_{y}$. In Fig. 13(c), for example, the period of the ground state is 1 . These considerations are most easily seen in the fermion case. In this case the eigenstates are constructed by the product of one-body states. The energy of a one-body state is easily obtained as (see Fig. 14)

$$
-2 t\left\{\cos \left(\pi \frac{n_{x}}{L_{x}+1}\right)+\cos \left[2 \pi\left(\frac{n_{y}+\Phi}{L_{y}}\right)\right]\right\},
$$

where $n_{x}=1,2, \ldots, L_{x}$ and $n_{y}=1,2, \ldots, L_{y}$. A manybody state is obtained by filling these states up to the number of particles. The energies of the one-body states change continuously as (4.2) when $\Phi$ changes. If we start from ground state and change $\Phi$ from zero to 1 , the state continuously changes to one of the excited states. The schematic diagram of the situation is shown in Fig. 14. For general anyon cases, there are many level crossings due to these spectral flows.

In some cases, there is an energy gap when we change the central flux as shown in Figs. 13(a) and 13(c); however, we cannot get this type of energy gap for larger systems [for example, Fig. 13(b)]. We cannot make a systematic conclusion as to the existence of the energy gap.

There are also spectral flows which cause many level crossings when we change the statistics. We show such 
results in Figs. 15: (a) on the annulus and (b) on the board.

\section{Comparison with the mean-field treatment}

In this subsection we want to investigate the accuracy of the mean-field treatment. Several authors treat the anyon system by an analytical method in which the sta- tistical flux is replaced by a uniform magnetic field. ${ }^{8-13,15,17,22}$ We compare the exact solution for the finite system to the mean-field results. Here we summarize the mean-field treatment of the statistical flux. We consider $\theta$ statistics anyon with density $n$ as a fermion with flux tube $\pi-\theta$ and want to replace the system with a system of noninteracting free fermions with $2 \pi \Phi_{F}$ magnetic field. We find $\Phi_{F}$ by the condition that the aver- (a)

\section{$1 / 4$ statistics anyon $3 \times 3 / 4$ on annulus}

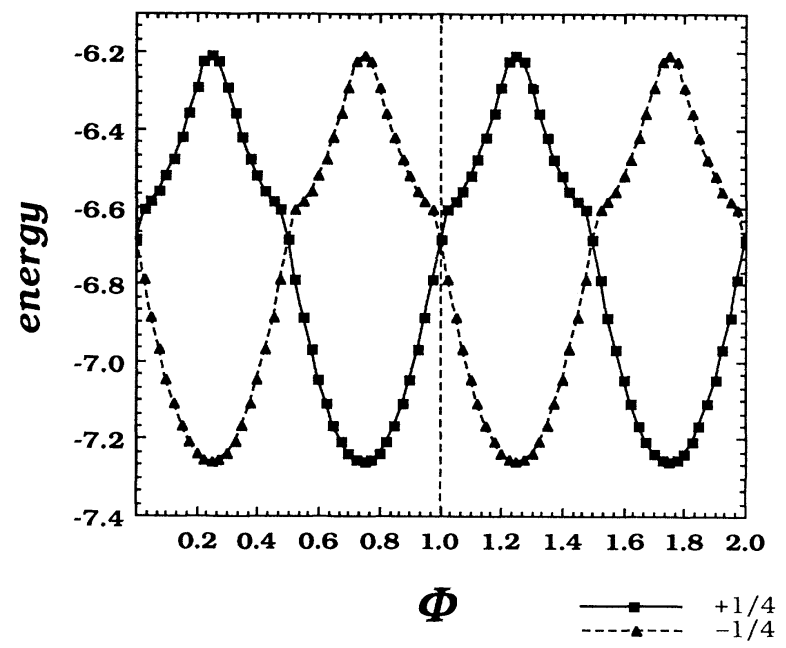

(b)

\section{1/5 statistics anyon $3 \times 3 / 4$ on annulus}

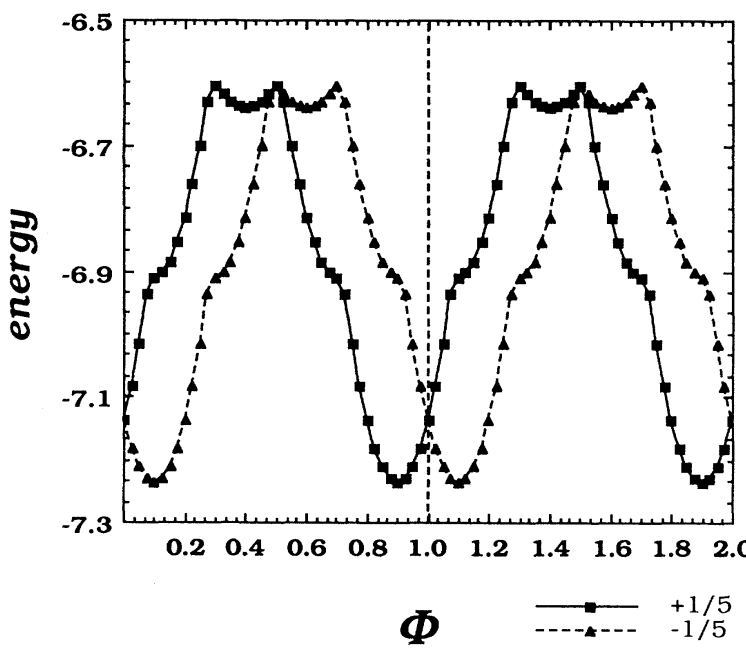

(c)

1/6 statistics anyon $3 \times 3 / 4$ on cylinder

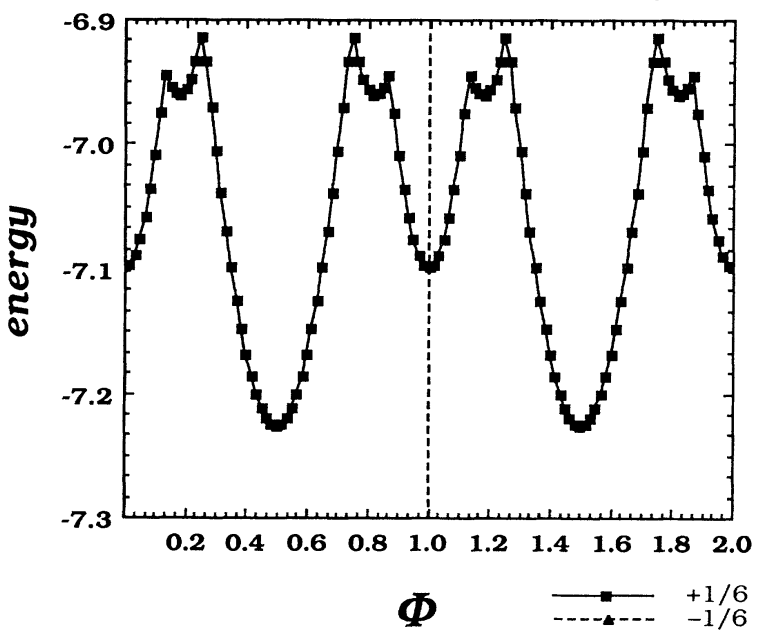

FIG. 12. Ground-state energy vs central flux diagrams for $\theta$ statistics and $-\theta$ statistics. (a) $2 \pi \frac{1}{4}$ statistics (semion) for a $3 \times 3 / 4$ annulus system, (b) $2 \pi \frac{1}{5}$ statistics for a $3 \times 3 / 4$ annulus system, (c) $2 \pi \frac{1}{6}$ statistics for a $3 \times 3 / 4$ cylinder system, (d) $2 \pi \frac{1}{10}$ statistics for a $3 \times 4 / 6$ cylinder system, and (e) $2 \pi \frac{1}{4}$ statistics (semion) for a $3 \times 3 / 3$ cylinder system. The condition $\exp [i 2(N-1)]=1$ is satisfied for (c), (d), and (e). In the (c), (d), and (e) cases, the curves for $+\theta$ and $-\theta$ coincide with each other and they are symmetric with respect to $\Phi=0$. 
(d)

$$
\text { 1/10 statistics anyon } 3 \times 4 / 6 \text { on cylinder }
$$

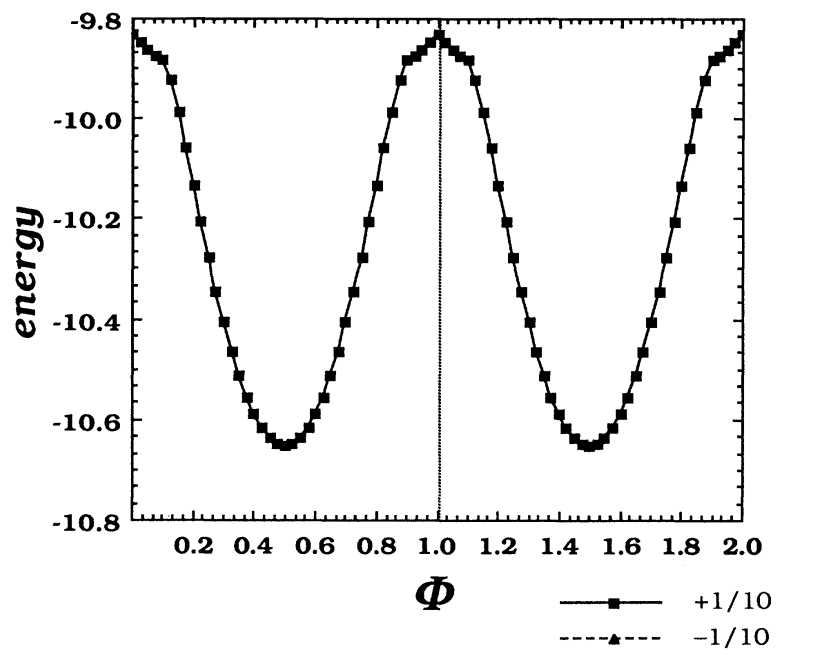

(e)

$1 / 4$ statistics anyon $3 \times 3 / 3$ on cylinder

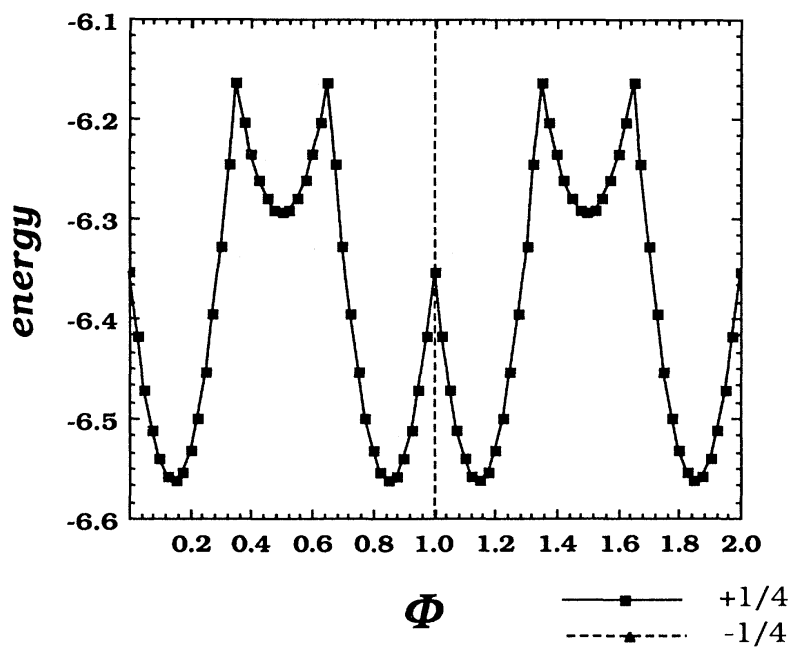

FIG. 12. (Continued).

aged Aharonov-Bohm phase of the particles to be the same as the phase due to the statistics. When one anyon moves around another anyon, the total change of the phase is $2(\pi-\theta)$. Thus the anyon gets a phase change $2(\pi-\theta) n S$ on average when it moves around an area $S$. If the particle is a fermion in a magnetic field, the change is $2 \pi \Phi_{F} S$. By this consideration one finds that the mean field is given by (see Fig. 16).

$$
\Phi_{F}=\frac{\pi-\theta}{\pi} n \text {. }
$$

In our finite-size calculation we also calculate a mean field which corresponds to $n^{\prime}=(N-1) / L_{x} L_{y}$ instead of $n=N / L_{x} L_{y}$. The factor $N-1$ is present because the anyon do not feel its own field at least in our lattice model (no self-interaction).

In Fig. 17(a) we show the exact ground-state energies for a $3 \times 4 / 5$ annulus system and the mean-field groundstate energies where the mean-field calculation is done by(4.3) with $n^{\prime}=(N-1) / L_{x} L_{y}$. It shows that the meanfield energy of the ground state agrees with that of the exact one within $5 \%$ until about semion statistics. There are many level crossings when we change the statistics and the global structure of these level crossings is also present in the mean-field calculation. In Fig. 17(b), we show the overlap between the ground-state wave function of the exact anyon system and that of the fermion system in the uniform magnetic field where $\Phi_{\text {boson }}$ is a strength of the mean field which corresponds to the boson system. It shows that the overlap between the exact ground state and that of the mean-field calculation is not so large if the statistics is less than about $0.9 \pi$. We can only say that the mean-field wave function is not so bad unless some level crossing occurs when the statistics changes from the fermion point. However, there are many level crossings and the region where the mean field is very good is not so large. In Fig. 18 we also show similar results for the fixed boundary condition. Figure 18(a) shows that the meanfield energy is very good unless level crossing occurs. We also show the result of the overlap in Fig. 18(b). It confirms that the mean-field calculation is good unless level crossing occurs. After the level crossing, however, the mean-field approximation is not so good. The range where the mean-field calculation is good is larger for the fixed boundary condition (board) compared to the periodic boundary condition (annulus).

We also perform a mean-field calculation from hardcore bosons. The hard-core bosons are not so easy to handle by the analytical method. Some authors, however, make such an approximation using a vortex structure. $^{23,24}$ In Figs. 19(a) and 19(b) we show the comparison of the energies by the mean-field from the hardcore-boson point for both the cylinder case and the board case. The mean-field strength is obtained by replacing $\pi$ $\theta$ by $\theta$ in (4.3), i.e., one has

$$
\Phi_{B}=\frac{\theta}{\pi} n
$$

We also show that the mean-field calculation using $n^{\prime}=(N-1) / L_{x} L_{y}$. The results also show that mean field is good unless level crossing occurs. The region where the mean field is good is wider than that of the mean-field calculation from the fermion, because there are fewer level crossings near the hard-core-boson point. 
(a)

$1 / 5$ statistics anyon $3 \times 3 / 4$ spectral flow

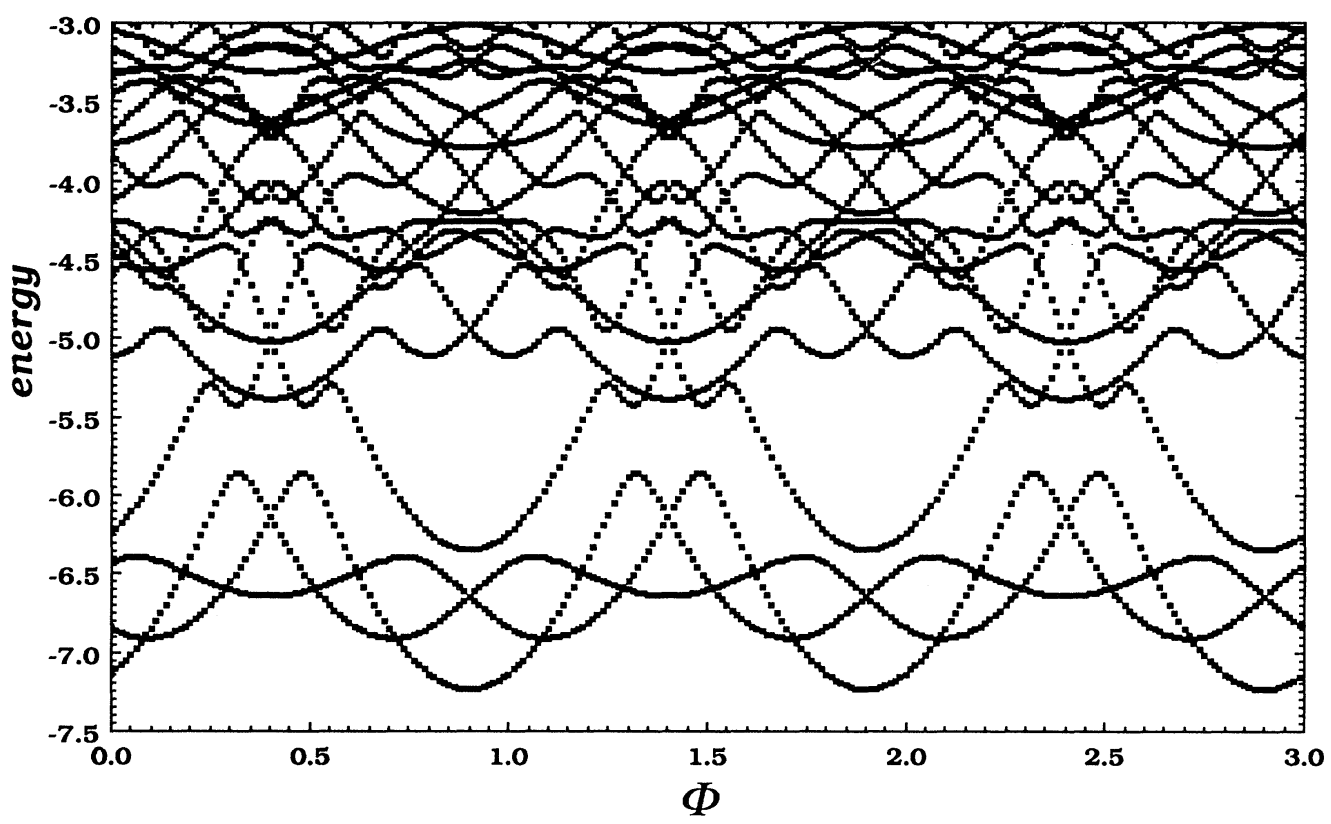

(b)

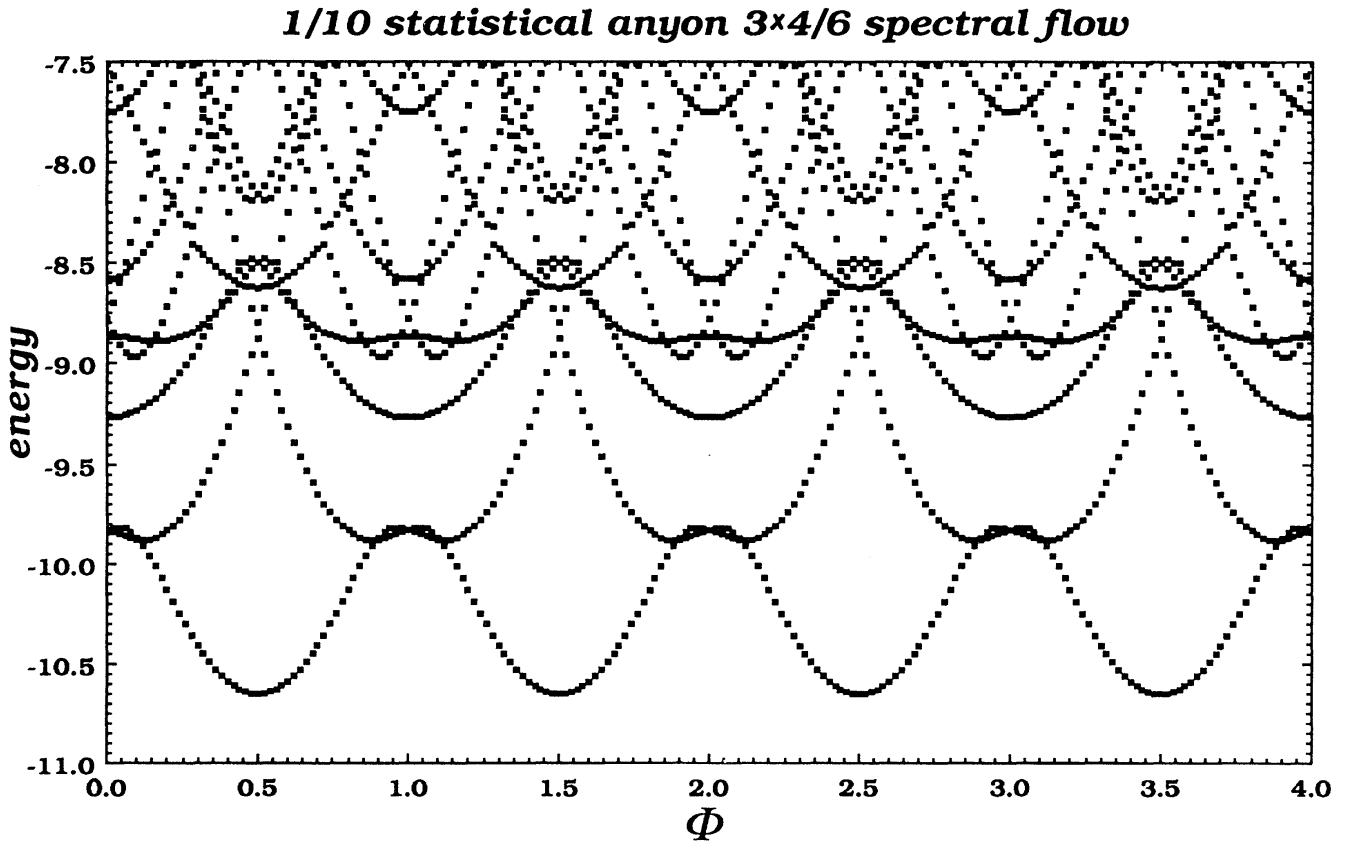

FIG. 13. Energies for the several lowest states as a function of the central flux: (a) $2 \pi \frac{1}{5}$ statistics for a $3 \times 3 / 4$ cylinder system, (b) $2 \pi \frac{1}{10}$ statistics for a $3 \times 4 / 6$ cylinder system, (c) $2 \pi \frac{1}{10}$ statistics for a $3 \times 3 / 3$ cylinder system, and (d) $2 \pi \frac{1}{4}$ statistics (semion) for a $3 \times 3 / 3$ cylinder system. In (a), the period of the lowest three states is not one but three. This shows the spectral flow clearly. Further there is an energy gap between these three states and the others. 
(c)

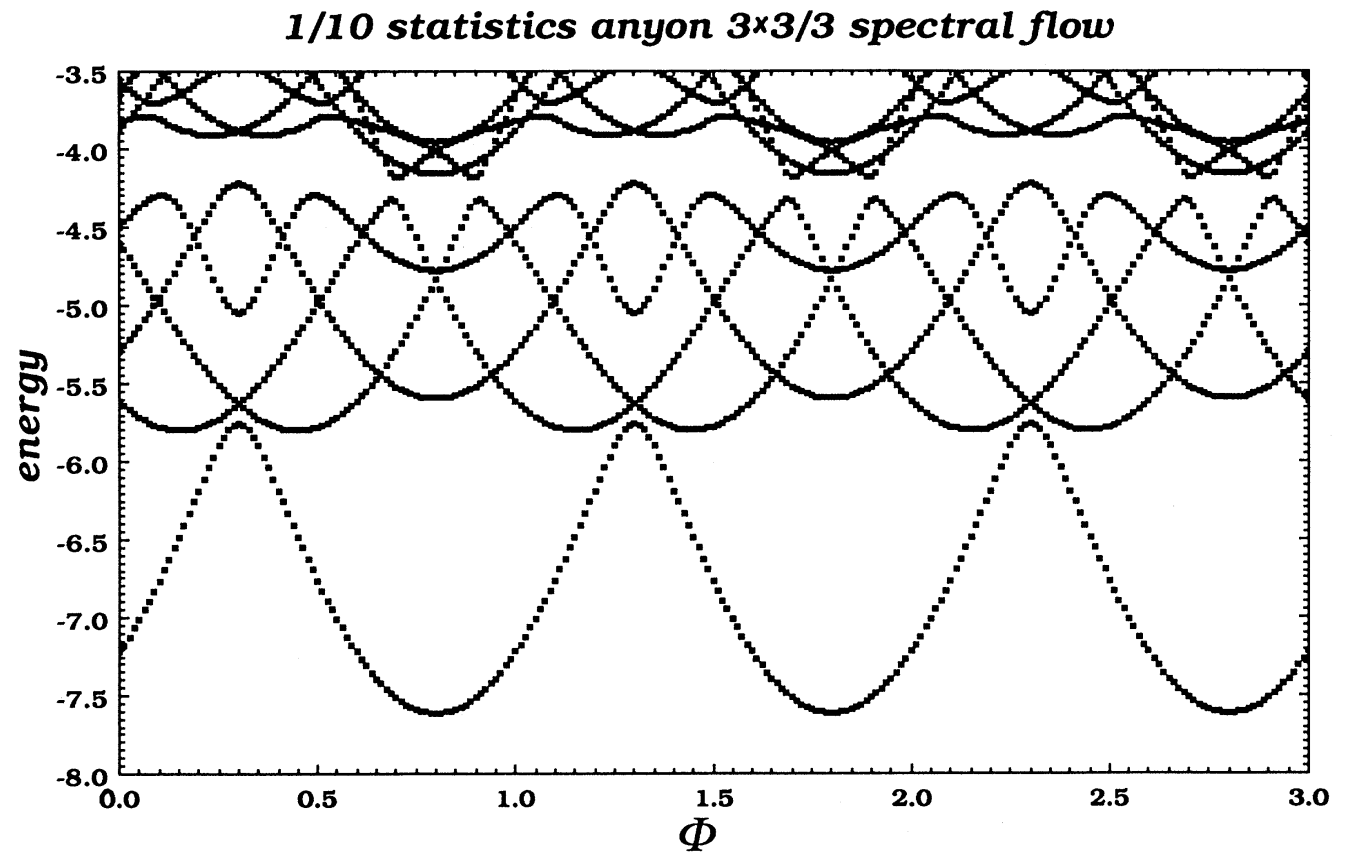

(d)

$1 / 4$ statistics anyon $3 \times 3 / 3$ spectral flow

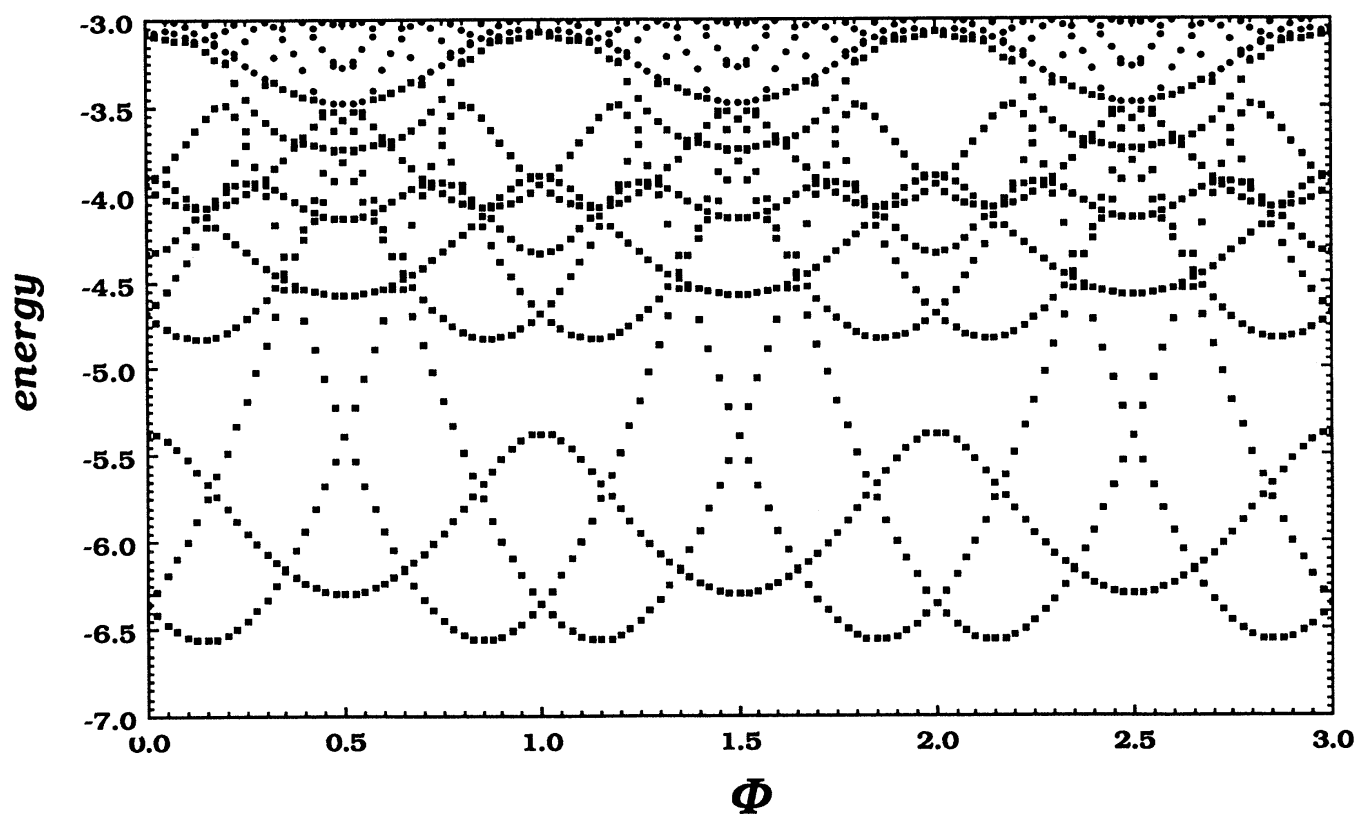

FIG. 13. (Continued). 


\section{SUMMARY AND DISCUSSIONS}

In this paper we presented a careful reexamination of anyons on a cylinder (or annulus), starting from the braid-group analysis. We focused on the topological features arising from the existence of noncontractible loops. In the usual two-dimensional plane, generators of the braid group are given by local exchanges of two neighboring particles which characterize the statistics of the system. We notice, however, that the cylinder, though noncompact, is still topologically nontrivial and differs from a board or a plane in that there are noncontractible loops on it. On the cylinder we find that there are additional generators besides the usual local exchanges in the braid group which correspond to moving anions around noncontractible loops. To define the representation of the braid group on the cylinder, we have to include the effect of the central flux $\Phi$ through the central hole. The braid group is characterized by two parameters $(\theta, \Phi)$ and these two are tangled with each other. We have to treat it carefully to obtain effects of the physical central flux $\Phi$ separately.

There is no ambiguity when we put anyons on the board. However, the rule for putting anyons on a square lattice has to be modified when the periodic boundary condition is imposed on one direction (cylinder). We have explicitly constructed two seemingly different rules to define anyons on a cylinder which satisfy the require-

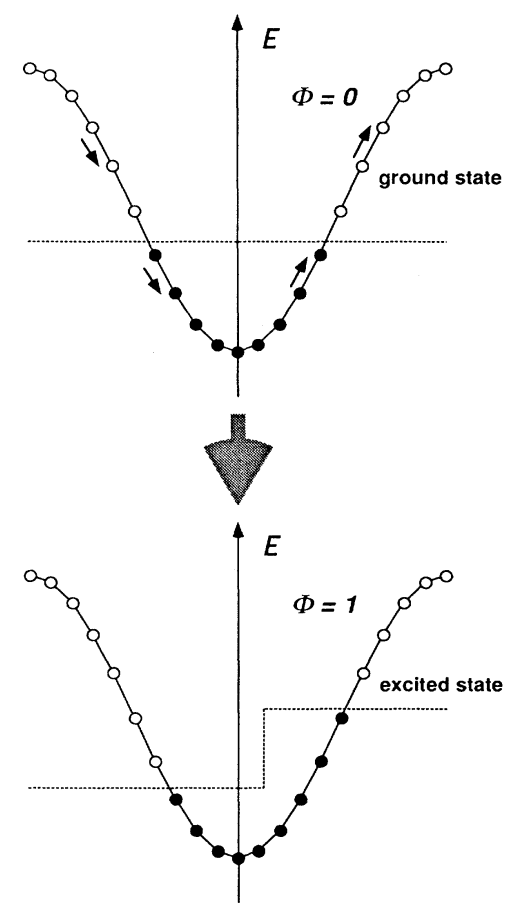

FIG. 14. Schematic diagram to explain the spectral flow of the free fermion. The ground state for $\Phi=0$ case is continuously connected to the first excited state for the $\Phi=1$. Notice that the $\Phi=1$ flux is removed from the Hamiltonian by the "large" gauge transformation (see text). ment of the braid group.

To check our analysis we have performed some finitesystem calculations. The consistency of our results has been verified by the agreement of the numerical results from our two different sets of rules. Although they both satisfy the braid-group requirements, the equivalence between them otherwise is not obvious at all. We have also explicitly shown that both of our rules reproduce the usual Fock space of fermion system.

(a)

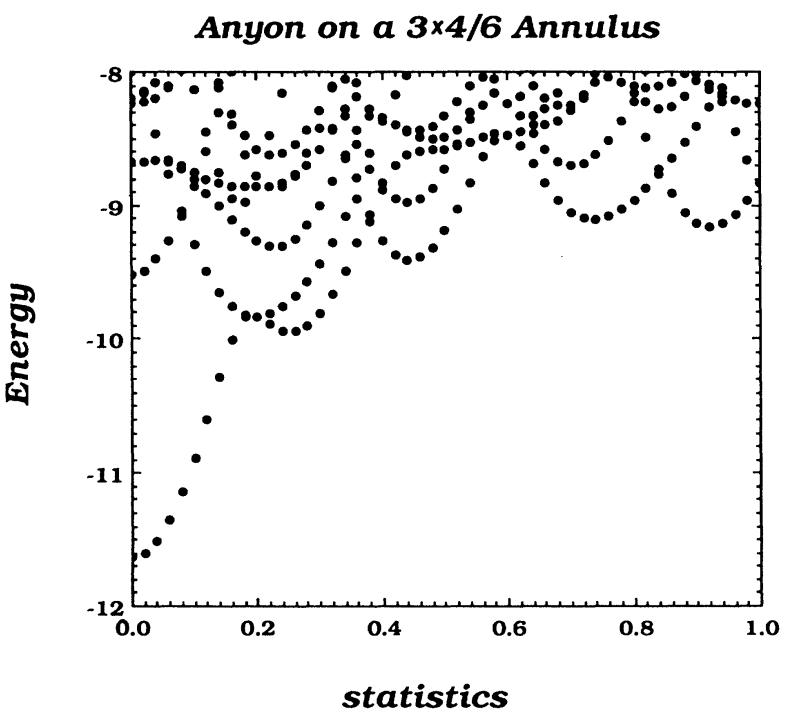

(b)

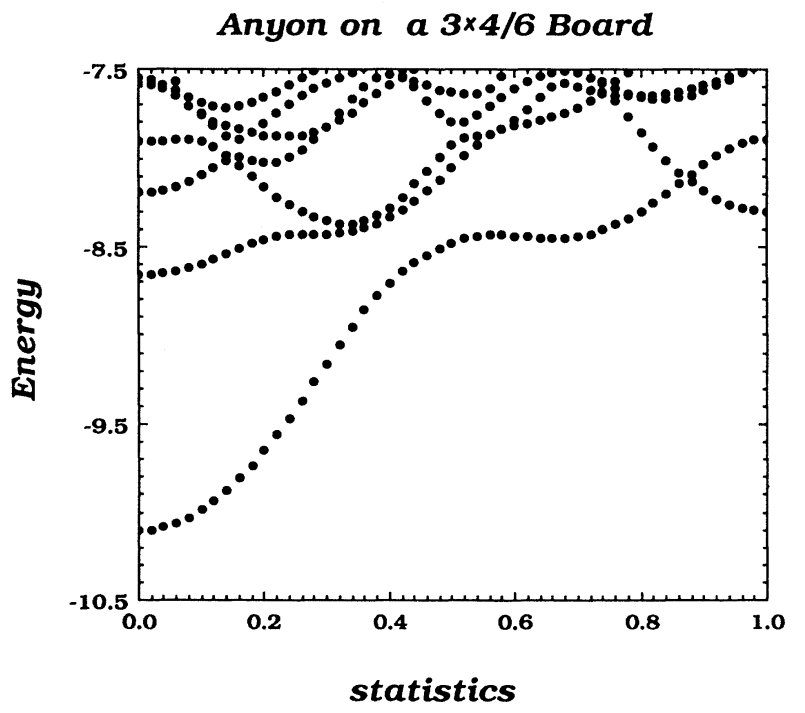

FIG. 15. Energies for the several lowest states as a function of the statistics: (a) for a $3 \times 4 / 6$ annulus system and (b) for a $3 \times 4 / 6$ board system. 


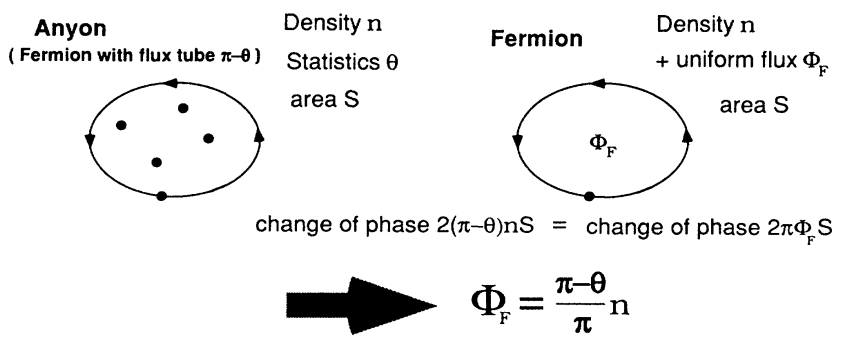

FIG. 16. Schematic diagram to explain the mean-field approximation.

(a)

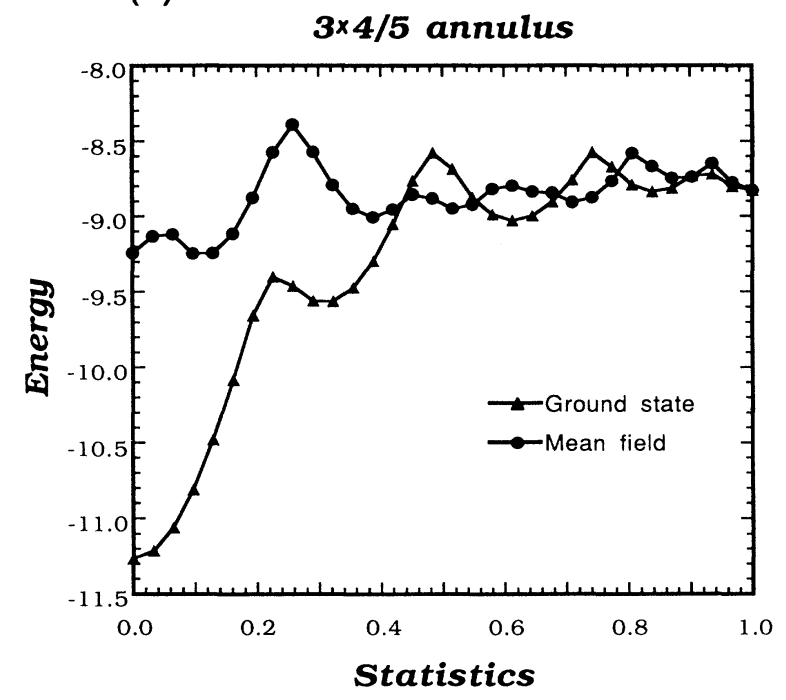

(b)

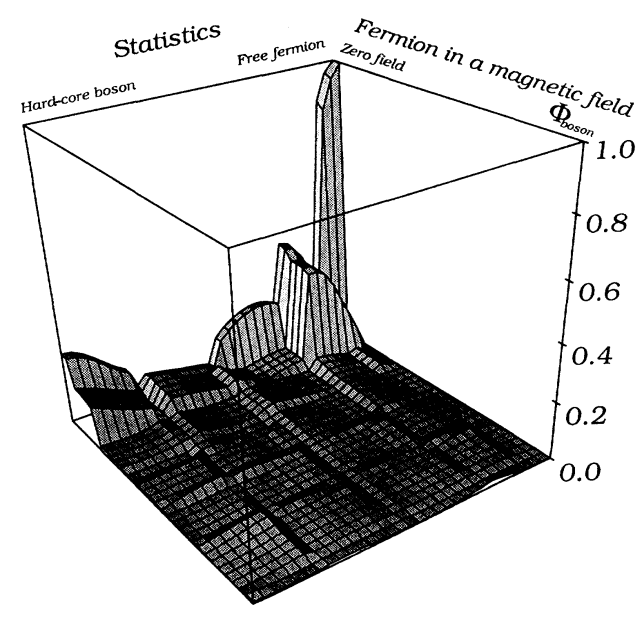

FIG. 17. (a) Comparison of the ground-state energies between the exact calculation and the mean-field calculation for a $3 \times 4 / 5$ annulus system. (b) Square of the overlap between the exact anyon wave function and the fermion wave function with uniform magnetic field. The diagonal direction corresponds to the mean-field value.
We have calculated not only the ground state but also several excited states. We have also calculated the spectral flow of the excited states with varying statistics or flux and have seen a lot of level crossings, which seem to be a general feature of anyon systems.

The cylinder and the annulus are topologically equivalent. However, they are not equivalent if we define anyons on them. We can define $\theta$ statistics anyons on a cylinder if and only if the condition $\exp [i 2 \theta(N-1)]=1$ holds, where $N$ is the number of the particles. Any $\theta$, however, is allowed on the annulus. At these special values of the statistics noted above, we can identify the

(a)

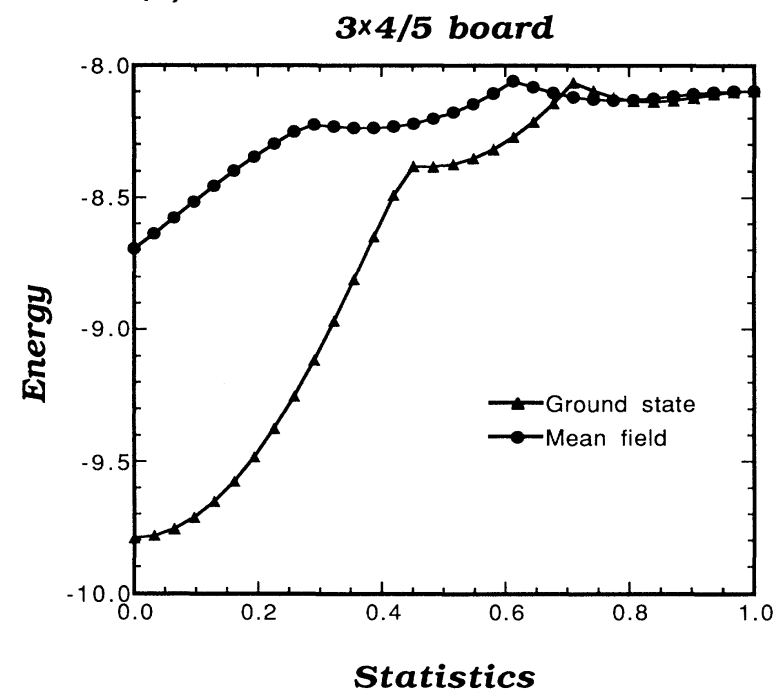

(b)

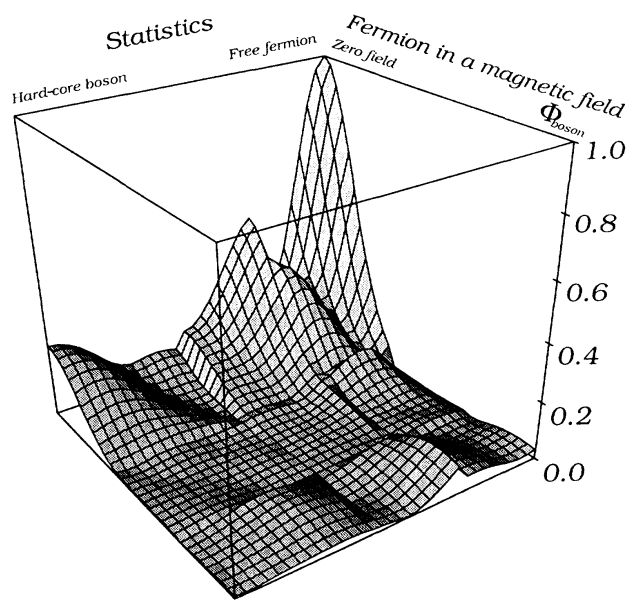

FIG. 18. (a) Comparison of the ground-state energies between the exact calculation and the mean-field calculation for a $3 \times 4 / 5$ board system. (b) Overlap between the exact anyon wave function and the fermion wave function with uniform magnetic field. The diagonal direction corresponds to the mean-field value. 
annulus as a cylinder. In general, there is a symmetry for the ground-state energy, that is, $E_{\text {annulus }}(\theta, \Phi)=E_{\text {annulus }}(-\theta,-\Phi)$; however, generically $E_{\text {annulus }}(\theta, \Phi) \neq E_{\text {annulus }}(-\theta, \Phi)$. If the above condition is satisfied

$E_{\text {cylinder }}(\theta, \Phi)=E_{\text {cylinder }}(-\theta, \Phi)=E_{\text {cylinder }}(\theta,-\Phi)$. This means that the direction of the fluxes (both central flux and virtual statistical flux) does not have any physical role in the cylinder geometry. We numerically checked these symmetries.

Finally, we have compared the energy and the wave function of the exact state with those of the mean-field calculation in the annulus (we cannot say "cylinder"). There are many level crossings when we change the statistics on the annulus. First we have performed a mean-field calculation from a free fermion. The energies are accurate in a few percent up to the semion point.

(a)

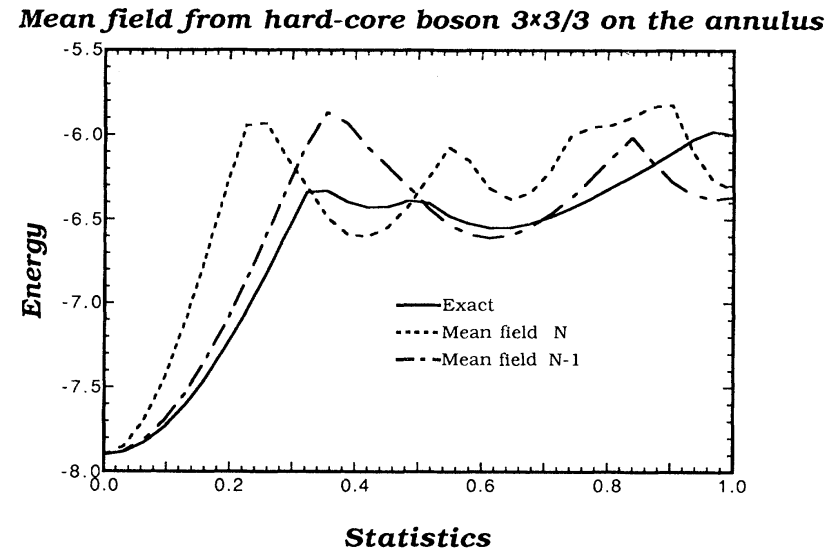

(b)

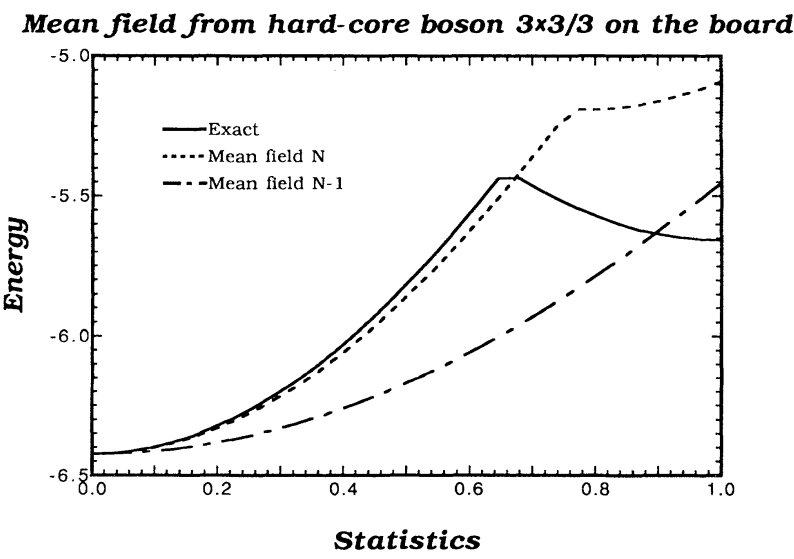

FIG. 19. Comparison of the ground-state energies between the exact calculation and the mean-field calculation for $3 \times 3 / 3$ cylinder system. In this case the particles are hard-core boson, that is, the mean field are from the hard-core boson point: (a) for a cylinder and (b) for a board.
However, if we compare the overlap of the wave function, the mean field is not so good if level crossing occurs. In this point of view, the region where the mean field is good is not so large away from the fermion point. We also calculated a similar calculation for a board geometry. The results are similar and the range is wider where the mean field is good. We have also compared a similar mean-field calculation from a hard-core boson. The tendency is similar to the mean-field calculation from the fermion. However, it seems that the level crossing does not occur near the hard-core-boson point and the range where the mean field is good is rather wider than that of the mean-field calculation from the free fermion.

Note added in proof. After the present work was completed, Chih-Han Sah proved that our defining relation of the braid group on the annulus is complete. ${ }^{25}$

\section{ACKNOWLEDGMENTS}

We thank G. S. Canright for explaining their gauge choice in their work. Y. S. Wu thanks the Japan Society for Promotion of Science for financial support which made his visit to Japan and this collaboration possible. His work was also supported in part by U. S. National Science Foundation through Grant No. PHY-8706501.

\section{APPENDIX}

Here, we give an intuitive derivation of rule $B$. We investigate the process in which the particles at $P$ hops to $Q$ in Fig. 7 under the periodic boundary condition. This process is decomposed into two steps. The first step is expressed by a solid line in Fig. 7(a). The change of the phase is given by

$$
\mathcal{N}(C) \theta,
$$

where $\mathcal{N}(C)$ is the number of the particles in region $C$. After this step, the configuration of the strings is given by Fig. 7(b) with string I. This string configuration is not a standard one and we have to deform it to the standard string II. The change of the phase in this step is given by

$$
[\mathcal{N}(A)+\mathcal{N}(C)+\mathcal{N}(D)+\mathcal{N}(E)] \theta .
$$

Thus the total change of the phase is

$$
[\mathcal{N}(A)+2 \mathcal{N}(C)+\mathcal{N}(D)+\mathcal{N}(E)] \theta .
$$

Using a simple constraint

$N=\mathcal{N}(A)+\mathcal{N}(B)+\mathcal{N}(C)+\mathcal{N}(D)+\mathcal{N}(E)+1$.

The total change of phase in this process is rewritten as

$$
(N-1) \theta+[\mathcal{N}(C)-\mathcal{N}(B)] \theta,
$$

where $[\mathcal{N}(C)-\mathcal{N}(B)] \theta$ could be given by rule $\mathrm{A}$, however, there is another factor $(N-1) \theta$. We have to give an extra factor $e^{i(N-1) \theta}$ to the simple rule $\mathrm{A}$ if some particle at the edge hops to the other edge under the periodic boundary condition. 
${ }^{1}$ J. M. Leinaas and J. Myrheim, Nuovo Cimento 37B, 1 (1977).

${ }^{2}$ F. Wilczek, Phys. Rev. Lett. 49, 957 (1982).

${ }^{3}$ Y. S. Wu, Phys. Rev. Lett. 52, 2103 (1984). The path-integral formulation on a multiply connected space is also found, in M. G. G. Laidlaw and C. M. DeWitt, Phys. Rev. D 3, 1275 (1975); L. S. Schulman, Techniques and Applications of Path Integration, (Wiley, New York, 1981).

${ }^{4}$ D. Arovas, J. R. Schrieffer, F. Wilczek, and A. Zee, Nucl. Phys. B251, [FS13], 117 (1985).

${ }^{5}$ B. I. Halperin, Phys. Rev. Lett. 52, 1583 (1984).

${ }^{6}$ D. Arovas, J. R. Schrieffer, and F. Wilczek, Phys. Rev. Lett. 53, 722 (1984).

${ }^{7}$ V. Kalmeyer and R. B. Laughlin, Phys. Rev. Lett. 59, 2095 (1987).

${ }^{8}$ R. B. Laughlin, Phys. Rev. Lett. 60, 2677 (1988).

${ }^{9}$ R. B. Laughlin, Science 242, 525 (1988).

${ }^{10}$ A. L. Fetter, C. B. Hanna, and R. B. Laughlin, Phys. Rev. B 39, 9679 (1989).

${ }^{11}$ C. B. Hanna, R. B. Laughlin, and A. L. Fetter, Phys. Rev. B 40, 8745 (1989).

${ }^{12}$ Y. -H. Chen, F. Wilczek, E. Witten, and B. I. Halperin, Int. J.
Mod. Phys. B3, 1001 (1989).

${ }^{13}$ Y. Kitazawa and H. Murayama, Phys. Rev. B 41, 11101 (1990).

${ }^{14}$ E. Fradkin, Phys. Rev. Lett. 63, 322 (1989), and (unpublished).

${ }^{15}$ G. S. Canright, S. M. Girvin, and A. Brass, Phys. Rev. Lett. 63, 2291 (1989).

${ }^{16}$ G. S. Canright, S. M. Girvin, and A. Brass, Phys. Rev. Lett. 63, 2295 (1989).

${ }^{17}$ G. S. Canright, S. M. Girvin, Int. J. Mod. Phys. B3, 1943 (1989).

${ }^{18}$ X. G. Wen, E. Dagaotto, and E. Fradkin, Phys. Rev. B 42, 6110 (1990).

${ }^{19}$ J. S. Birman, Commun. Pure Appl. Math. 22, 41 (1969).

${ }^{20}$ T. Einarsson, Phys. Rev. Lett. 64, 1995 (1990).

${ }^{21}$ D. J. Thouless and Y. S. Wu, Phys. Rev. B 31, 1191 (1985).

${ }^{22}$ M. D. Johnson and G. S. Canright, Phys. Rev. B 41, 6870 (1990).

${ }^{23}$ D. H. Lee and M. P. A. Fisher, Phys. Rev. Lett. 63, 903 (1989).

${ }^{24}$ X. G. Wen and A. Zee, Phys. Rev. B 41, 240 (1990).

${ }^{25}$ Chih-Han Sah (unpublished). 